\title{
LATE-TIME/STIFF-RELAXATION ASYMPTOTIC-PRESERVING APPROXIMATIONS OF HYPERBOLIC EQUATIONS
}

\author{
CHRISTOPHE BERTHON, PHILIPPE G. LeFLOCH, AND RODOLPHE TURPAULT
}

\begin{abstract}
We investigate the late-time asymptotic behavior of solutions to nonlinear hyperbolic systems of conservation laws containing stiff relaxation terms. First, we introduce a Chapman-Enskog-type asymptotic expansion and derive an effective system of equations describing the late-time/stiff-relaxation singular limit. The structure of this new system is discussed and the role of a mathematical entropy is emphasized. Second, we propose a new finite volume discretization which, in late-time asymptotics, allows us to recover a discrete version of the same effective asymptotic system. This is achieved provided we suitably discretize the relaxation term in a way that depends on a matrix-valued free-parameter, chosen so that the desired asymptotic behavior is obtained. Our results are illustrated with several models of interest in continuum physics, and numerical experiments demonstrate the relevance of the proposed theory and numerical strategy.
\end{abstract}

\section{INTRODUCTION}

Motivations. We are interested in the numerical approximation of solutions to nonlinear hyperbolic systems of conservation laws containing stiff-relaxation terms. An extensive literature is available on such systems since they arise in many physical problems of interest, for instance, in the modeling of complex multiphase flows involving phase transitions or kinetic-type phenomena. (See, for instance, 12, 13, 14, 22, 30.) Stiff-relaxation source terms are essential to model phenomena involving distinct physical time-scales. The derivation and the analysis (existence, stability) of an effective system of equations (also of hyperbolic type as the original system) as the relaxation time goes to zero is required for understanding the behavior of general solutions. (See, for instance, 25, 31, 34, 35.)

In the present paper, we go beyond these classical works and investigate the late-time behavior of solutions to systems with stiff-relaxation and, specifically, we consider the class of systems $(\varepsilon>0)$,

$$
\varepsilon \partial_{t} U+\partial_{x} F(U)=-\frac{R(U)}{\varepsilon}, \quad t>0, x \in \mathbb{R},
$$

where the main unknown $U: \mathbb{R} \times \mathbb{R}^{+} \rightarrow \Omega$ takes its values in a convex set $\Omega \subset$ $\mathbb{R}^{N}$. The associated homogeneous first-order system — obtained by neglecting the relaxation term $R: \Omega \rightarrow \mathbb{R}^{N}$ in the right-hand side of (1.1) - is assumed to be

Received by the editor November 15, 2010 and, in revised form, March 10, 2011; September $18,2011$.

2010 Mathematics Subject Classification. Primary 35L65, 65M99.

Key words and phrases. Nonlinear hyperbolic system, stiff source term, late-time behavior, diffusive regime, finite volume scheme, asymptotic preserving. 
hyperbolic in the sense that the $N \times N$ matrix $A:=D_{U} F$ admits real eigenvalues and a full basis of eigenvectors.

In comparison with classical works on this subject (see [11 as well as [1, 4, 10, 25]), the main novelty in the present work lies in the fact that the term $\varepsilon \partial_{t} U$ is rescaled to be proportional to $\varepsilon$. When $\varepsilon$ tends to zero, this will lead us to limit solutions whose behavior is quite distinct from those of standard relaxation limits. Indeed, as we establish below, the effective problem associated with (1.1) turns out to be a diffusion problem, in which the diffusion operator is determined by the (nonlinear) relaxation term $R$, in a way explicitly determined in the present work 1 . The relaxation map $R: \Omega \rightarrow \mathbb{R}^{N}$ is assumed to be sufficiently regular and satisfy the conditions introduced by Chen, Levermore, and Liu [1] for the "hyperbolic to hyperbolic" relaxation problem.

First, we assume the existence of a (constant) $n \times N$ matrix $Q$ with (maximal) rank $n<N$ such that

$$
Q R(U)=0, \quad U \in \Omega .
$$

From this, it follows that, if $U$ is a solution to (1.1), then $Q U: \mathbb{R} \times \mathbb{R}^{+} \rightarrow \mathbb{R}^{N}$ satisfies the system of $n$ conservation laws

$$
\varepsilon \partial_{t}(Q U)+\partial_{x}(Q F(U))=0
$$

in which the unknown takes values in the (convex) set

$$
\omega:=Q \Omega \subset \mathbb{R}^{n} .
$$

We also assume that a map $\mathcal{E}: \omega \rightarrow \Omega$ uniquely determines local equilibria, as defined by the relations

$$
Q \mathcal{E}(u)=u, \quad R(\mathcal{E}(u))=0, \quad u \in \omega .
$$

This suggests introducing the equilibrium manifold

$$
\mathcal{M}:=\{U=\mathcal{E}(u)\} .
$$

The dimension of the null space of the $N \times N$ matrix $B:=D R$ with the equilibrium submanifold is assumed to be "maximal" in the sense that

$$
\begin{aligned}
\operatorname{dim}(\operatorname{ker}(B(\mathcal{E}(u)))) & =n, \\
\operatorname{ker}(B(\mathcal{E}(u))) \cap \operatorname{Im}(B(\mathcal{E}(u))) & =\{0\} .
\end{aligned}
$$

Finally, since we are interested in the late-time behavior of solutions, it is necessary to impose that

$$
Q F(\mathcal{E}(u))=c, \quad u \in \omega,
$$

for some constant vector $c \in \mathbb{R}^{n}$. Indeed, in view of (1.3), we can formally write

$$
\varepsilon \partial_{t} u+\partial_{x} Q F(\mathcal{E}(u)) \rightarrow 0,
$$

so that $Q F(\mathcal{E}(u))$ must be a constant, normalized so that

$$
Q F=0 \quad \text { on } \mathcal{M} \text {. }
$$

\footnotetext{
${ }^{1}$ After this paper was completed, P. Marcati pointed out to the authors the relevant references 15] 16] in which several convergence results are established.
} 
Main results. Our objective in this paper is two-fold. First, we determine an effective system of equations driving the late-time asymptotic behavior of general solutions to (1.1) in the singular regime $\varepsilon \rightarrow 0$. Second, we introduce a novel numerical strategy allowing us to precisely recover the expected asymptotic behavior.

The paper is organized as follows. Section 2 is devoted to the asymptotic analysis: we derive an effective system and, under the hypotheses stated in this introduction as well as the assumption of the existence of a mathematical entropy compatible with the relaxation source term (in a sense specified below), we study the structure of this system which is found to be of diffusion-type and we show that the associated total entropy is nonincreasing in time.

Then, in Section 3, we generalize our analysis to a nonlinear version of (1.1), since this is required to encompass certain models arising in the aplications when the relaxation has strong nonlinearities. The class of systems under consideration in this section reads

$$
\varepsilon \partial_{t} U+\partial_{x} F(U)=-\frac{R(U)}{\varepsilon^{m}},
$$

where the parameter $m \geq 1$ introduces an additional scale in the problem. By imposing that

$$
R(\mathcal{E}(u)+\varepsilon U)=\varepsilon^{m} R(\mathcal{E}(u)+M U), \quad U \in \Omega, \quad u \in \omega,
$$

plus certain conditions on the $N \times N$ matrix $M$ (see Section 3), we derive again an effective system which, now, consists of nonlinear diffusion equations.

Next, in Section 4, we discuss several specific examples of practical interest and show that they fit into our general framework: (1) the Euler equations of compressible fluids with friction term, (2) the so-called $M 1$-model of radiative transfer, (3) a new model that couples the former two models and, finally, (4) the shallow-water system with nonlinear friction. Interestingly, the latter system does require the more general formalism (1.8).

In Section 5, we turn our attention to the discretization of (1.1) and (1.8). Recall that the "hyperbolic to hyperbolic" relaxation problem was treated numerically first by Jin and Xin [22]; our objective is to treat here the "hyperbolic to parabolic" relaxation problem. We introduce a new Godunov-type finite volume scheme which incorporates a suitable discretization of the source term and allows us to recover the expected asymptotic regime. The discrete form of the source term is derived by modifying a Riemann solver associated with the homogeneous system. The proposed numerical strategy is proven to satisfy a domain invariant principle in the sense that all of the computed states belong to the convex set $\Omega$. Finally, in Section 6, we conclude with several numerical experiments with the proposed asymptotic-preserving schemes, and we demonstrate the interest of our method on several of the physical models.

\section{LATE-TIME/STIFF-RELAXATION FRAMEWORK}

Derivation of the effective system. Throughout this section, we consider the nonlinear system of balance laws (1.1), and we impose the conditions stated in the introduction.

Our objective is to exhibit the system of effective equations satisfied by local equilibria $u=u(t, x) \in \omega$. In the spirit of Chapman-Enskog expansions of the 
kinetic theory [8], we consider a formal expansion of solutions $U$ to (1.1) in the form:

$$
U^{\varepsilon}=\mathcal{E}(u)+\varepsilon U_{1}+\varepsilon^{2} U_{2}+\ldots,
$$

where $U_{i}$ is referred to as the $\mathrm{i}^{\text {th }}$-order corrector. Such an expansion is natural in view of the assumptions (1.4) and (1.7). We consider the system

$$
\begin{aligned}
& \varepsilon \partial_{t} U^{\varepsilon}+\partial_{x} F\left(U^{\varepsilon}\right)=-\frac{1}{\varepsilon} R\left(U^{\varepsilon}\right), \\
& Q U^{\varepsilon}=u,
\end{aligned}
$$

in which we plug in the formal expansion (2.1) and match together terms of the same order of magnitude in $\varepsilon$.

From $Q U^{\varepsilon}=u$ we deduce that $Q \mathcal{E}(u)=u$, and $Q U_{i}=0$ for all $\mathrm{i}^{\text {th }}$-order correctors. Next, for sufficiently regular flux $F$, we obtain the following expansion:

$$
\begin{aligned}
F\left(U^{\varepsilon}\right)= & F(\mathcal{E}(u))+\varepsilon A(\mathcal{E}(u)) U_{1}+\frac{\varepsilon^{2}}{2} D_{U}^{2} F(\mathcal{E}(u))\left(U_{1}, U_{1}\right) \\
& +\varepsilon^{2} A(\mathcal{E}(u)) U_{2}+\mathcal{O}\left(\varepsilon^{3}\right) .
\end{aligned}
$$

Analogously, it is useful to write down the formal expansion of the relaxation term. Taking (1.4) into account, we obtain

$$
\frac{1}{\varepsilon} R\left(U^{\varepsilon}\right)=B(\mathcal{E}(u)) U_{1}+\frac{\varepsilon}{2} D_{U}^{2} R(\mathcal{E}(u)) .\left(U_{1}, U_{1}\right)+\varepsilon B(\mathcal{E}(u)) U_{2}+\mathcal{O}\left(\varepsilon^{2}\right) .
$$

We then plug (2.3) and (2.4) into (2.2) and obtain

$$
\begin{aligned}
& \varepsilon \partial_{t} \mathcal{E}(u)+\partial_{x} F(\mathcal{E}(u))+\varepsilon \partial_{x} A(\mathcal{E}(u)) U_{1} \\
& =-B(\mathcal{E}(u)) U_{1}-\frac{\varepsilon}{2} D_{U}^{2} R(\mathcal{E}(u)) .\left(U_{1}, U_{1}\right)-\varepsilon B(\mathcal{E}(u)) U_{2}+\mathcal{O}\left(\varepsilon^{2}\right) .
\end{aligned}
$$

Let us consider (2.5) and expand in $\varepsilon$. The zeroth-order terms give

$$
\partial_{x} F(\mathcal{E}(u))=-B(\mathcal{E}(u)) U_{1} .
$$

At this stage, we can solve for $U_{1}$ by recalling that $Q U_{1}=0$. Indeed, since we have imposed the properties (1.5) and (1.6) on the null space of $B(\mathcal{E}(u))$, then, for all fixed $J \in \mathbb{R}^{N}$, the system

$$
\begin{aligned}
& B(\mathcal{E}(u)) \cdot V=J, \\
& Q V=0,
\end{aligned}
$$

admits a unique solution $V \in \mathbb{R}^{n}$ if and only if $Q J=0$. (See Lemma 2.4 at the end of this section.) Here, we have $J=-\partial_{x} F(\mathcal{E}(u))$ which satisfies $Q \partial_{x} F(\mathcal{E}(u))=0$ by (1.7). As a consequence, for all $u \in \omega$, we can uniquely determine $U_{1}$ such that

$$
\begin{aligned}
& B(\mathcal{E}(u)) \cdot U_{1}=-\partial_{x} F(\mathcal{E}(u)), \\
& Q U_{1}=0 .
\end{aligned}
$$

Next, we consider the first-order terms in (2.5), and obtain

$$
\partial_{t} \mathcal{E}(u)+\partial_{x} A(\mathcal{E}(u)) U_{1}=-\frac{1}{2} D_{U}^{2} R(\mathcal{E}(u)) .\left(U_{1}, U_{1}\right)-B(\mathcal{E}(u)) U_{2} .
$$

Multiplying by $Q$ and using the assumption (1.2), we obtain

$$
Q D_{U}^{2} R(\mathcal{E}(u)) .\left(U_{1}, U_{1}\right) \equiv 0, \quad Q B(\mathcal{E}(u)) U_{2} \equiv 0 .
$$


Since $Q \mathcal{E}(u)=u$, we arrive at an effective system for the limit $u$, that is,

$$
\partial_{t} u=-\partial_{x}\left(Q A(\mathcal{E}(u)) U_{1}\right),
$$

where $U_{1}$ is the unique solution to (2.7).

The role of a mathematical entropy. Now, we assume the existence of a sufficiently regular, mathematical entropy $\Phi: \Omega \rightarrow \mathbb{R}$ so that the matrix $D_{U}^{2} \Phi(U) A(U)$ is symmetric for all $U$ in $\Omega$ and there exists an entropy-flux map $\Psi: \Omega \rightarrow \mathbb{R}$ such that

$$
D_{U} \Phi(U) A(U)=D_{U} \Psi(U), \quad U \in \Omega .
$$

Hence, all smooth solutions to (2.2) satisfy

$$
\varepsilon \partial_{t} \Phi\left(U^{\varepsilon}\right)+\partial_{x} \Psi\left(U^{\varepsilon}\right)=-\frac{1}{\varepsilon} D_{U} \Phi\left(U^{\varepsilon}\right) R\left(U^{\varepsilon}\right) .
$$

As usual, we impose $\Phi$ to be convex by requiring the $N \times N$ matrix $D_{U}^{2} \Phi(U)$ to be positive. In addition, we assume that the entropy is compatible with the relaxation in the sense that, for some map $\nu: \mathcal{M} \rightarrow \mathbb{R}^{n}$,

$$
\begin{aligned}
& D_{U} \Phi(U) R(U) \geq 0, \quad U \in \Omega, \\
& D_{U} \Phi=\nu Q \quad \text { on } \mathcal{M} .
\end{aligned}
$$

We now analyze the nature of the limiting system (2.8).

Theorem 2.1. Consider the nonlinear system of balance laws (1.1), under the assumptions (1.2)-(1.7) and (2.11)-(2.12). Then, the associated limiting system (2.8) takes the form

$$
\partial_{t} u=\partial_{x}\left(S \mathcal{L}^{-1}(u) S^{T}\left(\partial_{x} D_{u} \Phi(\mathcal{E}(u))\right)^{T}\right),
$$

where

$$
S:=Q A(\mathcal{E}(u))
$$

and

$$
\mathcal{L}(u):=D_{U}^{2} \Phi(\mathcal{E}(u)) B(\mathcal{E}(u)),
$$

and, for all $b \in \mathbb{R}^{N}$ with $Q b=0, \mathcal{L}^{-1}(u) . b$ denotes the unique solution to the system

$$
\mathcal{L}(u) . V=b, \quad Q V=0 .
$$

In addition, this system is dissipative with respect to the entropy $\Phi$ in the sense that the following positivity condition holds for all $u$ in $\omega$ :

$$
\left(\partial_{x} D_{u} \Phi(\mathcal{E}(u))\right) S \mathcal{L}^{-1}(u) S^{T}\left(\partial_{x} D_{u} \Phi(\mathcal{E}(u))\right)^{T} \geq 0 .
$$

Proof. We follow the strategy in [11] which we adapt to our problem. We first establish (2.13). To simplify the notation, we set

$$
\mathcal{D}(u)=-Q A(\mathcal{E}(u)) U_{1},
$$

to restate (2.8) as follows:

$$
\partial_{t} u=\partial_{x} \mathcal{D}(u) .
$$

Here, the vector $U_{1} \in \mathbb{R}^{N}$ is the unique solution of (2.7). Since $D_{U}^{2} \Phi(U)$ is a positive $N \times N$ matrix for all $U \in \Omega$, we can rewrite (2.7) as follows:

$$
\begin{aligned}
& D_{U}^{2} \Phi(\mathcal{E}(u)) B(\mathcal{E}(u)) \cdot U_{1}=-D_{U}^{2} \Phi(\mathcal{E}(u)) \partial_{x} F(\mathcal{E}(u)), \\
& Q U_{1}=0 .
\end{aligned}
$$


Invoking (2.15), the state vector $U_{1}$ is defined as the unique solution of

$$
\begin{aligned}
& \mathcal{L}(u) U_{1}=-D_{U}^{2} \Phi(\mathcal{E}(u)) \partial_{x} F(\mathcal{E}(u)), \\
& Q U_{1}=0 .
\end{aligned}
$$

As a consequence, by definition of $\mathcal{L}^{-1}(u)$ we have

$$
U_{1}=-\mathcal{L}^{-1}(u) D_{U}^{2} \Phi(\mathcal{E}(u)) \partial_{x} F(\mathcal{E}(u)) .
$$

From (2.14), we find

$$
\mathcal{D}(u)=S \mathcal{L}^{-1}(u) D_{U}^{2} \Phi(\mathcal{E}(u)) \partial_{x} F(\mathcal{E}(u)) .
$$

To obtain (2.13), we then establish

$$
D_{U}^{2} \Phi(\mathcal{E}(u)) \partial_{x} F(\mathcal{E}(u))=S^{T} v,
$$

where we define $v \in \mathbb{R}^{n}$ as follows:

$$
v=\left(\partial_{x} D_{u} \Phi(\mathcal{E}(u))\right)^{T} .
$$

To this end we differentiate (2.12) (which involves the map $\nu$ ) and we obtain

$$
\left(D_{u} \mathcal{E}(u)\right)^{T} D_{U}^{2} \Phi(\mathcal{E}(u))=D_{u}^{2} \Phi(\mathcal{E}(u)) Q .
$$

By transposition, we thus get

$$
D_{U}^{2} \Phi(\mathcal{E}(u)) D_{u} \mathcal{E}(u)=Q^{T} D_{U}^{2} \Phi(\mathcal{E}(u)) .
$$

Now, we have

$$
D_{U}^{2} \Phi(\mathcal{E}(u)) \partial_{x} F(\mathcal{E}(u))=D_{U}^{2} \Phi(\mathcal{E}(u)) A(\mathcal{E}(u)) D_{u} \mathcal{E}(u) \partial_{x} u
$$

Since the matrix $D_{U}^{2} \Phi(\mathcal{E}(u)) A(\mathcal{E}(u))$ is symmetric, we can write

$$
D_{U}^{2} \Phi(\mathcal{E}(u)) \partial_{x} F(\mathcal{E}(u))=(A(\mathcal{E}(u)))^{T} D_{U}^{2} \Phi(\mathcal{E}(u)) D_{u} \mathcal{E}(u) \partial_{x} u .
$$

We use (2.19) and obtain

$$
\begin{aligned}
D_{U}^{2} \Phi(\mathcal{E}(u)) \partial_{x} F(\mathcal{E}(u)) & =(A(\mathcal{E}(u)))^{T} Q^{T} D_{u}^{2} \Phi(\mathcal{E}(u)) \partial_{x} u \\
& =S^{T} v,
\end{aligned}
$$

and the identity (2.13) follows.

The proof will be completed as soon as we establish

$$
v^{T} S \mathcal{L}^{-1}(u) S^{T} v \geq 0
$$

which is equivalent to $v^{T} \mathcal{D}(u) \geq 0$ where $\mathcal{D}(u)=-S U_{1}$. We thus have

$$
v^{T} \mathcal{D}(u)=-v^{T} S U_{1}
$$

But from (2.16) and (2.17), we deduce

$$
v^{T} \mathcal{D}(u)=\left(D_{U}^{2} \Phi(\mathcal{E}(u)) B(\mathcal{E}(u)) U_{1}\right)^{T} U_{1} .
$$

As a consequence, the expected inequality $v^{T} \mathcal{D}(u) \geq 0$ will hold as soon as we prove that the matrix $D_{U}^{2} \Phi(\mathcal{E}(u)) D_{U} R(\mathcal{E}(u))$ is nonnegative.

Recalling the entropy assumption (2.11) and the equilibrium property $R(\mathcal{E}(u))=$ 0 , we have

$$
\begin{aligned}
& D_{U} \Phi(U) R(U) \geq 0, \quad U \in \Omega, \\
& \left.\left(D_{U} \Phi(U) R(U)\right)\right|_{U=\mathcal{E}(u)}=0, \quad u \in \omega .
\end{aligned}
$$


As a consequence, the matrix $\left.D_{U}^{2}\left(D_{U} \Phi(U) R(U)\right)\right|_{U=\mathcal{E}(u)}$ is nonnegative. Next, a calculation using the chain rule and the fact that $R$ vanishes on the equilibrium submanifold, that is,

$$
R(\mathcal{E}(u))=0
$$

leads us easily to

$$
\left.D_{U}^{2}\left(D_{U} \Phi(U) R(U)\right)\right|_{U=\mathcal{E}(u)}=D_{U}^{2} \Phi(\mathcal{E}(u)) B(\mathcal{E}(u))+\left(D_{U}^{2} \Phi(\mathcal{E}(u)) B(\mathcal{E}(u))\right)^{T},
$$

in which no third-order derivative terms arise since $R$ precisely vanishes on the equilibrium manifold. From the above identity, it then follows that

$$
\left(\left(D_{U}^{2} \Phi(\mathcal{E}(u)) B(\mathcal{E}(u))\right) U\right)^{T} U \geq 0, \quad U \in \Omega .
$$

We thus obtain the expected inequality $v^{T} \mathcal{D}(u) \geq 0$ and the proof is completed.

Monotonicity of the entropy. We then study the asymptotic behavior of the entropy inequality. In order to exhibit the entropy law satisfied by the equilibrium solution $\mathcal{E}(u)$, we need the following technical result.

Lemma 2.2. Under the assumptions of Theorem 2.1, the entropy-flux map restricted to the equilibrium $\Psi(\mathcal{E}(u))$ remains constant for all $u$ in $\omega$.

Proof. We consider the map $u \mapsto \Psi(\mathcal{E}(u))$ and, after differentiation, obtain

$$
D_{u} \Psi(\mathcal{E}(u))=D_{U} \Psi(\mathcal{E}(u)) D_{u} \mathcal{E}(u) .
$$

By definition of $\Psi$ given by (2.9), we have

$$
D_{u} \Psi(\mathcal{E}(u))=D_{U} \Phi(\mathcal{E}(u)) A(\mathcal{E}(u)) D_{u} \mathcal{E}(u) .
$$

Then, the assumption (2.12) made on $\Phi$ yields the following relation:

$$
\begin{aligned}
D_{u} \Psi(\mathcal{E}(u)) & =D_{u} \Phi(\mathcal{E}(u)) Q A(\mathcal{E}(u)) D_{u} \mathcal{E}(u), \\
& =D_{u} \Phi(\mathcal{E}(u)) D_{u} Q F(\mathcal{E}(u)) .
\end{aligned}
$$

Since we have $Q F(\mathcal{E}(u))=0$ over $\omega$, then $D_{u} Q F(\mathcal{E}(u))=0$. As a consequence, $D_{u} \Psi(\mathcal{E}(u))=0$ for all $u$ in $\omega$ and the proof is completed.

Equipped with this result, we can exhibit the asymptotic equation satisfied by the equilibrium entropy $\Phi(\mathcal{E}(u))$. Arguing the formal asymptotic expansion (2.1) satisfied by $U$ :

$$
U^{\varepsilon}=\mathcal{E}(u)+\varepsilon U_{1}+\ldots,
$$

where $U_{1}$ is defined as the unique solution to (2.7), we consider the formal expansion of each term in (2.10). First, since the entropy and entropy-flux are regular maps, we have

$$
\Phi\left(U^{\varepsilon}\right)=\Phi(\mathcal{E}(u))+\varepsilon D_{U} \Phi(\mathcal{E}(u)) U_{1}+\mathcal{O}\left(\varepsilon^{2}\right)
$$

and

$$
\Psi\left(U^{\varepsilon}\right)=\Psi(\mathcal{E}(u))+\varepsilon D_{U} \Psi(\mathcal{E}(u)) U_{1}+\mathcal{O}\left(\varepsilon^{2}\right) .
$$

But, by applying Lemma 2.2 we deduce the following relation:

$$
\partial_{x} \Psi\left(U^{\varepsilon}\right)=\varepsilon \partial_{x} D_{U} \Psi(\mathcal{E}(u)) U_{1}+\mathcal{O}\left(\varepsilon^{2}\right) .
$$

Similarly, concerning the entropy relaxation source term, we easily have

$$
D_{U} \Phi\left(U^{\varepsilon}\right) R\left(U^{\varepsilon}\right)=\varepsilon^{2} D_{U}^{2} \Phi(\mathcal{E}(u)) D_{U} R(\mathcal{E}(u)) U_{1}+\mathcal{O}\left(\varepsilon^{3}\right) .
$$


Now, we plug the expressions (2.23), (2.25), and (2.26) into (2.10), and consider the first-order terms only:

$$
\partial_{t} \Phi(\mathcal{E}(u))=-\partial_{x}\left(D_{U} \Psi(\mathcal{E}(u)) U_{1}\right)-U_{1}^{T}\left(D_{U}^{2} \Phi(\mathcal{E}(u)) B(\mathcal{E}(u))\right) U_{1},
$$

where, once again, $U_{1}$ is the unique solution to (2.7). From this entropy evolution law, we can state an entropy decreasing principle. Indeed, we have established that the matrix $D_{U}^{2} \Phi(\mathcal{E}(u)) D_{U} R(\mathcal{E}(u)$ ) is positive (cf. (2.21) and, as a consequence,

$$
U^{T}\left(D_{U}^{2} \Phi(\mathcal{E}(u)) B(\mathcal{E}(u))\right) U \geq 0, \quad U \in \Omega .
$$

We have thus proven the following statement.

Proposition 2.3. Under the assumptions of Theorem 2.1, the entropy is nonincreasing in the following sense:

$$
\partial_{t} \Phi(\mathcal{E}(u)) \leq-\partial_{x}\left(D_{U} \Psi(\mathcal{E}(u)) U_{1}\right) .
$$

To conclude this presentation of the asymptotic system of diffusion equations satisfied by stiff-relaxation terms for late times, let us emphasize the role played by the entropy. As recognized by Chen, Levermore, and Liu [11, the existence of a convex mathematical entropy provides an important structure to investigate the asymptotic regime satisfied by the model. However, the main discrepancy with [11] lies in the nature of the singular limit system. Indeed, in [11, the singular limit system turns out to be a hyperbolic system supplemented by an $\varepsilon$ first-order diffusive term. Here, the obtained asymptotic system defines a system of diffusion equations. Even if the limiting solution is smooth (due to the diffusive nature of the limiting system), the mathematical entropy is essential in order to establish the stability of the asymptotic regime.

A technical lemma. We end this section with a technical lemma that was useful in the above derivation of the asymptotic system.

Lemma 2.4. Let $A$ be a real $N \times N$ matrix such that $\operatorname{dim}(\operatorname{ker}(A))=n$ and $\operatorname{ker}(A) \cap$ $\operatorname{Im}(A)=\{0\}$. Also, let $Q$ be a real $n \times N$ matrix such that $\operatorname{rank}(Q)=n$ and $Q A=0$ Then, for any $b \in \mathbb{R}^{N}$, the linear system

$$
\begin{aligned}
& A x=b, \\
& Q x=0,
\end{aligned}
$$

admits a unique solution if and only if $Q b=0$.

Proof. If the system (2.28) has a solution, then left-multiplying $A x=b$ by $Q$ leads to $Q A x=Q b$. Since $Q A=0$, then $Q b=0$.

Now we suppose that $Q b=0$. Since $Q A=0$, then the columns of $Q^{T}$ are elements of the left null-space of $A$. Furthermore, $\operatorname{dim}\left(\operatorname{ker}\left(A^{T}\right)\right)=n=\operatorname{rank}(Q)$ therefore the columns of $Q^{T}$ are a basis of the left null-space of $A$. This implies that if $z_{0} \in \operatorname{ker}\left(A^{T}\right)$, then there exists $y_{0} \in \mathbb{R}^{n}$ such that $z_{0}=Q^{T} y_{0}$.

Let us consider $z \in \mathbb{R}^{N}$ such that $Q z=0$. Using the fundamental theorem of linear algebra we have $z=z_{0}+z_{1}$ where $z_{0} \in \operatorname{ker}\left(A^{T}\right)$ and $z_{1} \in \operatorname{Im}(A)$.

We then characterize $z_{0}$ and $z_{1}$ using their respective definitions: there exists $y_{1} \in$ $\mathbb{R}^{N}$ such that $z_{1}=A y_{1}$, and there exists $y_{0} \in \mathbb{R}^{n}$ such that $z_{0}=Q^{T} y_{0}$. With these characterizations we have

$$
0=Q z=Q z_{0}+Q z_{1}=Q Q^{T} y_{0}+Q A y_{1}=Q Q^{T} y_{0}+0
$$


since $Q A=0$. Now, $\operatorname{rank}(Q)=n$ implies that $Q Q^{T}$ is a symmetric positive-definite matrix and therefore that $y_{0}=0$. Therefore, $z \in \operatorname{Im}(A)$ if and only if $Q z=0$.

By considering first the existence issue, the above property on $b$ allows us to say that $b \in \operatorname{Im}(A)$ and hence there exists $x \in \mathbb{R}^{N}$ such that $A x=b$. If $x$ is such a solution and since $\operatorname{ker}(A) \oplus \operatorname{Im}(A)=\mathbb{R}^{N}$, we have $x=x_{0}+x_{1}$ where $x_{0} \in \operatorname{ker}(A)$ and $x_{1} \in \operatorname{Im}(A)$. With these notation, $x_{1}$ is a solution to (2.28).

Next, we consider next the uniqueness issue and suppose that $x_{1}$ and $x_{2}$ are two solutions to (2.28). Then $\left(x_{1}-x_{2}\right)$ is the solution to

$$
\begin{aligned}
& A\left(x_{1}-x_{2}\right)=0, \\
& Q\left(x_{1}-x_{2}\right)=0,
\end{aligned}
$$

and therefore $\left(x_{1}-x_{2}\right) \in \operatorname{ker}(A) \cap \operatorname{Im}(A)=\{0\}$. Hence, the solution to (2.28) is unique.

\section{Nonlinear Diffusive REgIME}

Derivation of a nonlinear asymptotic system. Some physical models involve several relaxation time-scales. More precisely, we suppose now that the ratio of the relaxation time and the late time under consideration is no longer constant. The extended model thus reads

$$
\varepsilon \partial_{t} U+\partial_{x} F(U)=-\frac{1}{\varepsilon^{m}} R(U), \quad t>0, \quad x \in \mathbb{R},
$$

with $U \in \Omega \subset \mathbb{R}^{N}$. Here, $m \geq 1$ denotes an integer. The case $m=1$ has been discussed in the previous section, and we now assume $m>1$.

Several of the assumptions made for $m=1$ are kept here. Precisely, we assume the existence of an $n \times N$ matrix $Q$ with rank $n<N$ satisfying (1.2), as well as the existence of a map $\mathcal{E}: \omega \rightarrow \Omega$ satisfying (1.4). We also impose that the flux $F$ satisfies (1.7). Concerning the nonlinear relaxation map $R$, an additional assumption must be imposed: there exists an $N \times N$ matrix, denoted $M(\varepsilon)$, such that

$$
R(\mathcal{E}(u)+\varepsilon U)=\varepsilon^{m} R(\mathcal{E}(u)+M(\varepsilon) U), \quad U \in \Omega, \quad u \in \omega .
$$

The matrix $M(\varepsilon)$ is assumed to be sufficiently smooth in $\varepsilon \in[0,1]$.

The assumption initially made on the kernel of the matrix $B(\mathcal{E}(u))$ is irrelevant if $m>1$. Indeed, this kernel assumption was imposed to ensure the existence and uniqueness of the solution to (2.6). We are going to see that this linear system is no longer relevant and must be replaced by a nonlinear problem. In this sense, the proposed extension is called nonlinear since the diffusive asymptotic regime will involve a nonlinear differential operator.

First, to derive the effective system of equations satisfied by the local equilibrium $u \in \omega$, we introduce again a Chapman-Enskog-type expansion:

$$
U^{\varepsilon}=\mathcal{E}(u)+\varepsilon U_{1}+\varepsilon^{2} U_{2}+\ldots
$$

We plug this expansion into (3.1) and match terms of the same order in $\varepsilon$.

Enforcing $Q U^{\varepsilon}=u$, the condition (1.4) on the local equilibrium implies $Q U_{i}=0$ for each corrector term. Note that the expansion (2.3) for the flux remains valid, and we only have to evaluate the expansion of the relaxation term by recalling (3.2). Indeed, this assumption gives

$$
R\left(U^{\varepsilon}\right)=\varepsilon^{m} R\left(\mathcal{E}(u)+M(\varepsilon) U_{1}+\varepsilon M(\varepsilon) U_{2}+\ldots\right),
$$


thus

$$
\begin{aligned}
\frac{1}{\varepsilon^{m}} R\left(U^{\varepsilon}\right)= & R\left(\mathcal{E}(u)+M(0) U_{1}\right)+\varepsilon B\left(M(0) U_{1}\right) \cdot\left(M(0) U_{2}\right) \\
& +\left.\varepsilon D_{\varepsilon}\left(M(\varepsilon) U_{1}\right)\right|_{\varepsilon=0} \cdot R\left(\mathcal{E}(u)+M(0) U_{1}\right)+\mathcal{O}\left(\varepsilon^{2}\right) .
\end{aligned}
$$

Setting (2.3) and (3.3) into (3.1), we obtain

$$
\begin{array}{rl}
\varepsilon \partial_{t} & \mathcal{E}(u)+\partial_{x} F(\mathcal{E}(u))+\varepsilon A(\mathcal{E}(u)) U_{1} \\
= & -R\left(\mathcal{E}(u)+M(0) U_{1}\right)-\varepsilon B\left(M(0) U_{1}\right) \cdot\left(M(0) U_{2}\right) \\
& -\left.\varepsilon D_{\varepsilon}\left(M(\varepsilon) U_{1}\right)\right|_{\varepsilon=0} \cdot R\left(\mathcal{E}(u)+M(0) U_{1}\right)+\mathcal{O}\left(\varepsilon^{2}\right) .
\end{array}
$$

Considering the zeroth-order terms, we get

$$
\partial_{x} F(\mathcal{E}(u))=-R\left(\mathcal{E}(u)+M(0) U_{1}\right),
$$

which turns out to be a nonlinear system of equations with unknown $U_{1}$, supplemented by the condition $Q U_{1}=0$.

At this level, we see that the assumption on the kernel of $B(\mathcal{E}(u))$ is no longer relevant (or sufficient) in order to solve (2.6), since we now have to consider

$$
\begin{aligned}
& R\left(\mathcal{E}(u)+M(0) U_{1}\right)=-\partial_{x} F(\mathcal{E}(u)), \\
& Q U_{1}=0 .
\end{aligned}
$$

In view of the strong nonlinearities involved in this equation, we tacitly assume the existence and uniqueness of the solution, denoted $\bar{U}_{1}$, of (3.5). In the applications, this property will be checked directly.

Then, we match first-order terms resulting from (3.4) to get

$$
\begin{aligned}
& \partial_{t} \mathcal{E}(u)+\partial_{x} A(\mathcal{E}(u)) \cdot \bar{U}_{1} \\
& =-B\left(M(0) \bar{U}_{1}\right) \cdot\left(M(0) U_{2}\right)-\left.D_{\varepsilon}\left(M(\varepsilon) \bar{U}_{1}\right)\right|_{\varepsilon=0} \cdot R\left(\mathcal{E}(u)+M(0) \bar{U}_{1}\right) .
\end{aligned}
$$

Since $Q \mathcal{E}(u)=u$ for all $u \in \omega$, and since $Q R(U)=0$ and $Q B(U)=0$ for all $U \in \Omega$, by multiplying the above relation by $Q$, we obtain

$$
\partial_{t} u=-\partial_{x}\left(Q A(\mathcal{E}(u)) \bar{U}_{1}\right) .
$$

Once again, this equilibrium equation involves a nonlinear differential operator in the right-hand side since $\bar{U}_{1}$ is a nonlinear map applied to first-order space derivatives.

Similarly to the "linear" case governed by (2.8), we want to interpret (3.6) as a system of diffusion equations. We thus assume the existence of a convex entropy $\Phi: \Omega \rightarrow \mathbb{R}$ which satisfies all the compatibility conditions imposed in the previous section. The matrix $D_{U}^{2} \Phi(U) D_{U} F(U)$ to be symmetric for all $U \in \Omega$ and, in addition, we assume that the compatibility conditions (2.11) and (2.12) hold. Smooth solutions to (3.1) satisfy the additional balance law

$$
\varepsilon \partial_{t} \Phi\left(U^{\varepsilon}\right)+\partial_{x} \Psi\left(U^{\varepsilon}\right)=-\frac{1}{\varepsilon^{m}} D_{U} \Phi\left(U^{\varepsilon}\right) R\left(U^{\varepsilon}\right) .
$$

Equipped with this convex entropy, we observe that $\bar{U}_{1}$ is, equivalently, a solution to the nonlinear algebraic system

$$
\begin{aligned}
& D_{U}^{2} \Phi(\mathcal{E}(u)) R\left(\mathcal{E}(u)+M(0) U_{1}\right)=-D_{U}^{2} \Phi(\mathcal{E}(u)) \partial_{x} F(\mathcal{E}(u)), \\
& Q U_{1}=0
\end{aligned}
$$

for any convex entropy compatible with the relaxation term. 
Next, consider the matrix $S$ defined by (2.14) and the vector $v$ given by (2.18). In view of (2.20), the above system is equivalent to

$$
\begin{aligned}
& D_{U}^{2} \Phi(\mathcal{E}(u)) R\left(\mathcal{E}(u)+M(0) U_{1}\right)=-S^{T} v, \\
& Q U_{1}=0 .
\end{aligned}
$$

With some abuse of notation and for the sake of clarity, we set

$$
\mathcal{N}_{u}\left(U_{1}\right)=D_{U}^{2} \Phi(\mathcal{E}(u)) R\left(\mathcal{E}(u)+M(0) U_{1}\right)
$$

and introduce the notation

$$
\bar{U}_{1}=\mathcal{N}_{u}^{-1}\left(-S^{T} v\right)
$$

Hence, the equilibrium equation (3.6) reads as follows:

$$
\partial_{t} u=\partial_{x}\left(-S \mathcal{N}_{u}^{-1}\left(-S^{T} v\right)\right) .
$$

Once again, we note the crucial role played by the convex entropies. Indeed, we now exhibit the limiting system of equations satisfied by the equilibrium entropy $\Phi(\mathcal{E}(u))$. We skip here the details of the computation which are similar to the linear case. Lemma 2.2 still holds, as well as the entropy expansion (2.23) and (2.25). In fact, only the entropy relaxation source term expansion changes. We now obtain

$$
D_{U} \Phi\left(U^{\varepsilon}\right) R\left(U^{\varepsilon}\right)=\varepsilon^{m+1} \bar{U}_{1}^{T} D_{U}^{2} \Phi(\mathcal{E}(u)) R\left(\mathcal{E}(u)+M(0) \bar{U}_{1}\right) .
$$

Plugging these expansions into (3.7) and considering first-order terms, we find

$$
\partial_{t} \Phi(\mathcal{E}(u))=-\partial_{x}\left(D_{U} \Psi(\mathcal{E}(u)) \bar{U}_{1}\right)-\bar{U}_{1}^{T} D_{U}^{2} \Phi(\mathcal{E}(u)) R\left(\mathcal{E}(u)+M(0) \bar{U}_{1}\right) .
$$

To conclude this section, we show that the asymptotic system of equations (3.10) is of diffusive-type, and that an associated mathematical entropy is nondecreasing.

Lemma 3.1. Let $\bar{U}_{1}$ be given by (3.5). Assume the existence of a nonnegative map $c(u) \geq 0$ such that

$$
R\left(\mathcal{E}(u)+M(0) \bar{U}_{1}\right)=c(u) \bar{U}_{1}, \quad u \in \omega .
$$

Then the limiting equation (3.6) is nonlinearly dissipative with respect to the entropy in the following sense:

$$
-v^{T} S \mathcal{N}_{u}^{-1}\left(-S^{T} v\right) \geq 0, \quad u \in \omega,
$$

where $S$ is given by (2.14) and $v$ by (2.18).

Moreover, the entropy is decreasing as follows:

$$
\partial_{t} \Phi(\mathcal{E}(u)) \leq-\partial_{x}\left(D_{U} \Psi(\mathcal{E}(u)) \cdot \bar{U}_{1}\right) .
$$

Proof. Arguing (3.8) and the definition (3.9), we have

$$
-v^{T} S \mathcal{N}_{u}^{-1}\left(-S^{T} v\right)=\bar{U}_{1}^{T} D_{U}^{2} \Phi(\mathcal{E}(u)) R\left(\mathcal{E}(u)+M(0) \bar{U}_{1}\right) .
$$

By recalling (3.12), we obtain

$$
-v^{T} S \mathcal{N}_{u}^{-1}\left(-S^{T} v\right)=c(u) \bar{U}_{1}^{T} D_{U}^{2} \Phi(\mathcal{E}(u)) \bar{U}_{1} .
$$

The inequality (3.13) follows from the convexity of the entropy. Recalling (3.11) we obtain (3.14), and the proof is completed. 


\section{Physical EXAmples}

Euler equations with friction term. Many models involving distinct physical scales enter the framework proposed in the present paper and, specifically, we will now illustrate the interest of our framework with four examples. We begin with the isentropic Euler equations supplemented with a friction term (cf. [27, 33] for further details). The asymptotics for this model has already been considered in the literarure and, more recently, relevant numerical techniques have been proposed [5, 9. (See also [26, 3.) Importantly, this model satisfies all of the conditions required in Section 3 , above.

The Euler model with friction reads

$$
\begin{aligned}
& \varepsilon \partial_{t} \rho+\partial_{x} \rho v=0, \\
& \varepsilon \partial_{t} \rho v+\partial_{x}\left(\rho v^{2}+p(\rho)\right)=-\frac{1}{\varepsilon} \rho v,
\end{aligned}
$$

where $\rho>0$ denotes the density and $v \in \mathbb{R}$ the velocity of a compressible fluid. The pressure function $p: \mathbb{R}^{+} \rightarrow \mathbb{R}^{+}$is assumed to be sufficiently regular and satisfy $p^{\prime}(\rho)>0$, so that the first-order homogeneous system associated with (4.1) is strictly hyperbolic.

In view of (1.1), we should set

$$
U=\left(\begin{array}{c}
\rho \\
\rho v
\end{array}\right), \quad F(U)=\left(\begin{array}{c}
\rho v \\
\rho v^{2}+p(\rho)
\end{array}\right), \quad R(U)=\left(\begin{array}{c}
0 \\
\rho v
\end{array}\right)
$$

which corresponds to the matrix

$$
Q=\left(\begin{array}{ll}
1 & 0
\end{array}\right)
$$

and scalar local equilibria $u=\rho$, with

$$
\mathcal{E}(u)=\left(\begin{array}{l}
\rho \\
0
\end{array}\right) .
$$

As required, we also have $Q F(\mathcal{E}(u))=0$, and it is easily checked that all our assumptions of the previous sections hold.

Considering now the asymptotic diffusive regime in the limit $\varepsilon \rightarrow 0$, we note that the equilibrium solution must satisfy (2.8), i.e.,

$$
\partial_{t} \rho=-\partial_{x}\left(Q A(\mathcal{E}(u)) U_{1}\right),
$$

where

$$
A(\mathcal{E}(u))=\left(\begin{array}{cc}
0 & 1 \\
p^{\prime}(\rho) & 0
\end{array}\right)
$$

and $U_{1}$ is the unique solution to (2.7). Since

$$
B(\mathcal{E}(u))=\left(\begin{array}{cc}
0 & 0 \\
0 & 1
\end{array}\right), \quad \partial_{x} F(\mathcal{E}(u))=\left(\begin{array}{c}
0 \\
\partial_{x} p(\rho)
\end{array}\right),
$$

the diffusive regime associated with this Euler model with friction is described by the equation

$$
\partial_{t} \rho=\partial_{x}^{2}(p(\rho)) .
$$

Based on Theorem 2.1. we observe that the diffusive nature of (4.3) follows from the existence of a convex entropy which is compatible with the relaxation source 
term in the sense of (2.11)-(2.12). Indeed, by introducing the internal energy $e(\rho)>0$ defined by

$$
e^{\prime}(\rho)=\frac{p(\rho)}{\rho^{2}},
$$

we see that smooth solutions to (4.1) satisfy

$$
\varepsilon \partial_{t}\left(\rho \frac{v^{2}}{2}+\rho e(\rho)\right)+\partial_{x}\left(\rho \frac{v^{2}}{2}+\rho e(\rho)+p(\rho)\right) v=-\frac{1}{\varepsilon} \rho v^{2} .
$$

The function $\Phi(U)=\rho \frac{v^{2}}{2}+\rho e(\rho)$ is a convex entropy satisfying the compatibility conditions with the relaxation terms.

The $M 1$ model for radiative transfer. The second example of interest relies on a more complex physical set-up, relevant in radiative transfer and referred to as the M1-model [17, 29]. (See also [2, 6, 7, 20].) This model reads

$$
\begin{aligned}
& \varepsilon \partial_{t} e+\partial_{x} f=\frac{1}{\varepsilon}\left(\tau^{4}-e\right), \\
& \varepsilon \partial_{t} f+\partial_{x}(\chi(f / e) e)=-\frac{1}{\varepsilon} f \\
& \varepsilon \partial_{t} \tau=\frac{1}{\varepsilon}\left(e-\tau^{4}\right),
\end{aligned}
$$

where $e>0$ is the radiative energy and $f$ the radiative flux, restricted by the "flux limitation" condition

$$
\left|\frac{f}{e}\right| \leq 1
$$

and $\tau>0$ denotes the temperature. The function $\chi:[-1,1] \rightarrow \mathbb{R}^{+}$stands for the Eddington factor defined by

$$
\chi(\xi)=\frac{3+4 \xi^{2}}{5+2 \sqrt{4-3 \xi^{2}}} .
$$

Once again, we rely on the notation introduced in the previous sections (cf. system (1.1) ) and we write

$$
U=\left(\begin{array}{c}
e \\
f \\
\tau
\end{array}\right), \quad F(U)=\left(\begin{array}{c}
f \\
\chi\left(\frac{f}{e}\right) e \\
0
\end{array}\right), \quad R(U)=\left(\begin{array}{c}
e-\tau^{4} \\
f \\
\tau^{4}-e
\end{array}\right)
$$

The local equilibria are described by the map

$$
\mathcal{E}(u)=\left(\begin{array}{c}
\tau^{4} \\
0 \\
\tau
\end{array}\right)
$$

where $u=\tau+\tau^{4}$ is now a scalar. We set $Q=\left(\begin{array}{lll}1 & 0 & 1\end{array}\right)$ and, in agreement with (1.7), we have $Q F(\mathcal{E}(u))=0$.

The asymptotic regime is governed by (2.8) and to exhibit its explicit formulation we need the expression of $A(\mathcal{E}(u))$ and $U_{1}$. A straightforward calculation gives

$$
A(\mathcal{E}(u))=\left(\begin{array}{ccc}
0 & 1 & 0 \\
\chi(0) & \chi^{\prime}(0) & 0 \\
0 & 0 & 0
\end{array}\right)=\left(\begin{array}{ccc}
0 & 1 & 0 \\
\frac{1}{3} & 0 & 0 \\
0 & 0 & 0
\end{array}\right)
$$


while $U_{1}$ is the solution to (2.7), which here reads

$$
\begin{gathered}
\left(\begin{array}{ccc}
1 & 0 & -4 \tau^{3} \\
0 & 1 & 0 \\
-1 & 0 & 4 \tau^{3}
\end{array}\right) U_{1}=\left(\begin{array}{c}
0 \\
\partial_{x}\left(\frac{\tau^{4}}{3}\right) \\
0
\end{array}\right) \\
\left(\begin{array}{lll}
1 & 0 & 1
\end{array}\right) U_{1}=0
\end{gathered}
$$

We thus obtain

$$
U_{1}=\left(\begin{array}{c}
0 \\
\frac{4}{3} \tau^{3} \partial_{x} \tau \\
0
\end{array}\right),
$$

and, according to (2.8), the asymptotic regime is governed by the following diffusion equation:

$$
\partial_{t}\left(\tau+\tau^{4}\right)=\partial_{x}\left(\frac{4}{3} \tau^{3} \partial_{x} \tau\right)
$$

A coupled Euler/M1 model. We propose here an example of a system that degenerates into a system of diffusion equations of dimension $n>1$. To do so, we couple the Euler model (4.1) with the $M 1$ model (4.4) as follows:

$$
\begin{aligned}
& \varepsilon \partial_{t} \rho+\partial_{x} \rho v=0, \\
& \varepsilon \partial_{t} \rho v+\partial_{x}\left(\rho v^{2}+p(\rho)\right)=-\frac{\kappa}{\varepsilon} \rho v+\frac{\sigma}{\varepsilon} f, \\
& \varepsilon \partial_{t} e+\partial_{x} f=0, \\
& \varepsilon \partial_{t} f+\partial_{x} \chi\left(\frac{f}{e}\right) e=-\frac{\sigma}{\varepsilon} f,
\end{aligned}
$$

in the notation previously introduced. Here, $\kappa$ and $\sigma$ denote positive constants. Even though this is a toy model, the pressure has to be sufficiently small in order to represent an application of physical interest. We will therefore consider the following pressure law:

$$
p(\rho)=C_{p} \rho^{\eta}, \quad C_{p} \ll 1, \quad \eta>1 .
$$

In the formula (1.1) we need to set

$$
U=\left(\begin{array}{c}
\rho \\
\rho v \\
e \\
f
\end{array}\right), \quad F(U)=\left(\begin{array}{c}
\rho v \\
\rho v^{2}+p(\rho) \\
f \\
\chi\left(\frac{f}{e}\right) e
\end{array}\right), \quad R(U)=\left(\begin{array}{c}
0 \\
\kappa \rho v-\sigma f \\
0 \\
\sigma f
\end{array}\right) .
$$

The local equilibrium is given by

$$
\mathcal{E}(u)=\left(\begin{array}{c}
\rho \\
0 \\
e \\
0
\end{array}\right), \quad u=Q U=\left(\begin{array}{c}
\rho \\
e
\end{array}\right), \quad Q=\left(\begin{array}{cccc}
1 & 0 & 0 & 0 \\
0 & 0 & 1 & 0
\end{array}\right) .
$$

Once again, one has $Q F(\mathcal{E}(u))=0$. To derive the asymptotic regime, we exhibit $A(\mathcal{E}(u))$ and $U_{1}$ :

$$
A(\mathcal{E}(u))=\left(\begin{array}{cccc}
0 & 1 & 0 & 0 \\
p^{\prime}(\rho) & 0 & 0 & 0 \\
0 & 0 & 0 & 1 \\
0 & 0 & \frac{1}{3} & 0
\end{array}\right), \quad U_{1}=\left(\begin{array}{c}
0 \\
\frac{1}{\kappa}\left[-\partial_{x} p(\rho)-\frac{1}{3} \partial_{x} e\right] \\
0 \\
-\frac{1}{3 \sigma} \partial_{x} e
\end{array}\right)
$$


The asymptotic diffusive regime of the system (4.5) is therefore given by

$$
\begin{aligned}
& \partial_{t} \rho-\frac{1}{\kappa} \partial_{x}^{2} p(\rho)-\frac{1}{3 \kappa} \partial_{x}^{2} e=0, \\
& \partial_{t} e-\frac{1}{3 \sigma} \partial_{x}^{2} e=0 .
\end{aligned}
$$

Shallow water with strong friction effect. The last suggested example is devoted to the well-known shallow-water model supplemented by a strong friction term in a late-time regime where the friction is assumed to dominate over the convection.

This model is given as follows:

$$
\begin{aligned}
& \varepsilon \partial_{t} h+\partial_{x} h v=0, \\
& \varepsilon \partial_{t} h v+\partial_{x}\left(h v^{2}+p(h)\right)=-\frac{\kappa^{2}(h)}{\varepsilon^{2}} g h v|h v|,
\end{aligned}
$$

where $h>0$ is the water height, $v \in \mathbb{R}$ the velocity, and $p(h)=g \frac{h^{2}}{2}$ the pressure law. Here, $g>0$ is the usual gravity constant, while the friction coefficient $\kappa: \mathbb{R}^{+} \rightarrow \mathbb{R}^{+}$ is a given and positive function. In 28, several examples of friction $\kappa$ are proposed. A standard choice is $\kappa(h)=\frac{\kappa_{0}}{h}$ where $\kappa_{0}>0$ is a given parameter.

We note that this model enters the framework of the nonlinear extension governed by (3.1) with $m=2$. According to the notation involved in (3.1), we have set

$$
U=\left(\begin{array}{c}
h \\
h v
\end{array}\right), \quad F(U)=\left(\begin{array}{c}
h v \\
h v^{2}+p(h)
\end{array}\right), \quad R(U)=\left(\begin{array}{c}
0 \\
\kappa^{2}(h) g h v|h v|
\end{array}\right) .
$$

Concerning the equilibrium, we have

$$
\mathcal{E}(u)=\left(\begin{array}{c}
h \\
0
\end{array}\right)
$$

where $u=h$ is a scalar and $Q=\left(\begin{array}{ll}1 & 0\end{array}\right)$. The assumption (3.2) easily holds since

$$
R(\mathcal{E}(u)+\varepsilon U)=\varepsilon^{2} R(\mathcal{E}(U)+M(\varepsilon) U),
$$

where

$$
M(\varepsilon)=\left(\begin{array}{cc}
\varepsilon & 0 \\
0 & 1
\end{array}\right) .
$$

We turn our attention now to the asymptotic regime which is governed by the nonlinear diffusion equation (3.6). To get its explicit form, we have to exhibit $U_{1}=(\alpha \beta)^{T}$, the solution to (3.5), which reads as follows:

$$
\begin{aligned}
& \alpha=0, \\
& \kappa^{2}(h) g \beta|\beta|=-\partial_{x} p(h) .
\end{aligned}
$$

We easily find that

$$
\beta=-\frac{\sqrt{h} \partial_{x} h}{\kappa(h) \sqrt{\left|\partial_{x} h\right|}},
$$

and the effective nonlinear diffusion equation is thus

$$
\partial_{t} h=\partial_{x}\left(\frac{\sqrt{h}}{\kappa(h)} \frac{\partial_{x} h}{\sqrt{\left|\partial_{x} h\right|}}\right) .
$$

This is a nonlinear Laplacian equation (for instance, see [23] and references therein). 
Lemma 3.1 applies here with the following entropy. By introducing the internal energy $e(h)=g h / 2$, we see that smooth solutions to (4.7) satisfy

$$
\varepsilon \partial_{t}\left(h \frac{v^{2}}{2}+g \frac{h^{2}}{2}\right)+\partial_{x}\left(h \frac{v^{2}}{2}+g h^{2}\right) v=-\frac{\kappa^{2}(h)}{\varepsilon^{2}} g h v^{2}|h v| .
$$

The entropy $\Phi(U)=h \frac{v^{2}}{2}+g \frac{h^{2}}{2}$ satisfies all the required properties, and the condition (3.12) holds since

$$
R\left(\mathcal{E}(u)+M(0) \bar{U}_{1}\right)=\left(\begin{array}{c}
0 \\
\partial_{x} p(h)
\end{array}\right)
$$

where $\bar{U}_{1}=(0 \beta)^{T}$. As a consequence, we obtain $R\left(\mathcal{E}(u)+M(0) \bar{U}_{1}\right)=c(u) \bar{U}_{1}$ with

$$
c(u)=g \kappa(h) \sqrt{h\left|\partial_{x} h\right|} \geq 0 .
$$

Hence, the limit equation (4.8) is of diffusive-type in the sense of Lemma 3.1

\section{Asymptotic-PRESERVing SCHEMES}

Objective. In this section, we consider the numerical approximation of solutions to (1.1). Our goal is to derive a class of numerical schemes that restore the relevant asymptotic regime, given by (2.8), in the limit $\varepsilon \rightarrow 0$. One of the main difficulties when deriving asymptotic-preserving schemes lies in the independent role played by each $\varepsilon$ and the mesh size. More precisely, the limit discrete diffusion equation (as $\varepsilon$ tends to zero) must be obtained independently of the space mesh size.

Such a numerical problem was investigated during the last decade on several specific examples. For instance, in [5], the Euler equations with friction term are considered. Relating works to radiative transfer and the $M 1$-model are given in [6, 20]. The reader is also referred to [3] where distinct physical applications are proposed.

In the present work, we propose a generalization of a numerical scheme derived to approximate the solutions to the Euler equations with friction and the $M 1$-model 3. To sketch this suggested numerical procedure, we first consider a standard finite volume method to approximate weak solutions to the homogeneous system associated with (1.1) in which we have omitted $\varepsilon$ :

$$
\partial_{t} U+\partial_{x} F(U)=0 .
$$

Next, we derive a suitable correction to obtain a finite volume discretization of the source term. Hence, the corrected finite volume method gives approximate solutions of the system

$$
\partial_{t} U+\partial_{x} F(U)=-\gamma R(U)
$$

where $\gamma>0$ is a fixed parameter. Finally, the asymptotic behavior of the scheme is analyzed. We consider a late-time compatible discretization and we fix $\gamma=\frac{1}{\varepsilon}$. The asymptotic scheme is thus obtained in the limit of $\varepsilon$ to zero. Modulo a suitable correction, it is thus proved to be asymptotic preserving.

A discretization of (5.2). As a first step, we suggest to consider the well-known Godunov-type scheme introduced by Harten, Lax, and van Leer 21] with a single constant intermediate state, to approximate the weak solutions to (5.1).

We consider a uniform mesh made of cells $\left[x_{i-1 / 2}, x_{i+1 / 2}\right)$ where $x_{i+1 / 2}=x_{i}+\frac{\Delta x}{2}$ for all $i \in \mathbb{Z}$ with a constant cell size $\Delta x$. The time discretization is defined by 
$t^{n+1}=t^{n}+\Delta t$ where the time increment will be restricted later on by a CFL-like condition.

We define the discrete initial data as follows:

$$
U^{0}(x)=\frac{1}{\Delta x} \int_{x_{i-1 / 2}}^{x_{i+1 / 2}} U(x, 0) d x, \quad x \in\left[x_{i-1 / 2}, x_{i+1 / 2}\right) .
$$

We seek a piecewise constant approximation of the exact solution of (5.1) at the time $t^{n}$,

$$
U^{n}(x)=U_{i}^{n}, \quad x \in\left[x_{i-1 / 2}, x_{i+1 / 2}\right),
$$

with $U_{i}^{n} \in \Omega$ for all $i \in \mathbb{Z}$.

By considering a suitable sequence of approximate Riemann solvers, we can evolve this approximation and get a piecewise constant function $\tilde{U}^{n+1}(x)$, which is an approximation of the solution to (5.1) at the time $t^{n}+\Delta t$. Following Harten, Lax, and van Leer [21, at each cell interface we use the following approximate Riemann solver:

$$
\tilde{U}_{\mathcal{R}}\left(\frac{x}{t} ; U_{L}, U_{R}\right)= \begin{cases}U_{L}, & \frac{x}{t}<-b, \\ \tilde{U}^{\star}, & -b<\frac{x}{t}<b, \\ U_{R}, & \frac{x}{t}>b,\end{cases}
$$

where $b>0$ is a fixed and sufficiently large constant, and

$$
\tilde{U}^{\star}=\frac{1}{2}\left(U_{L}+U_{R}\right)-\frac{1}{2 b}\left(F\left(U_{R}\right)-F\left(U_{L}\right)\right) .
$$

As a consequence, as soon as the following CFL restriction holds:

$$
b \frac{\Delta t}{\Delta x} \leq \frac{1}{2}
$$

we are considering a juxtaposition of noninteracting approximate Riemann solver (cf. Figure 1) denoted $\tilde{U}_{\Delta x}^{n}\left(x, t^{n}+t\right)$ for $t \in[0, \Delta t)$.

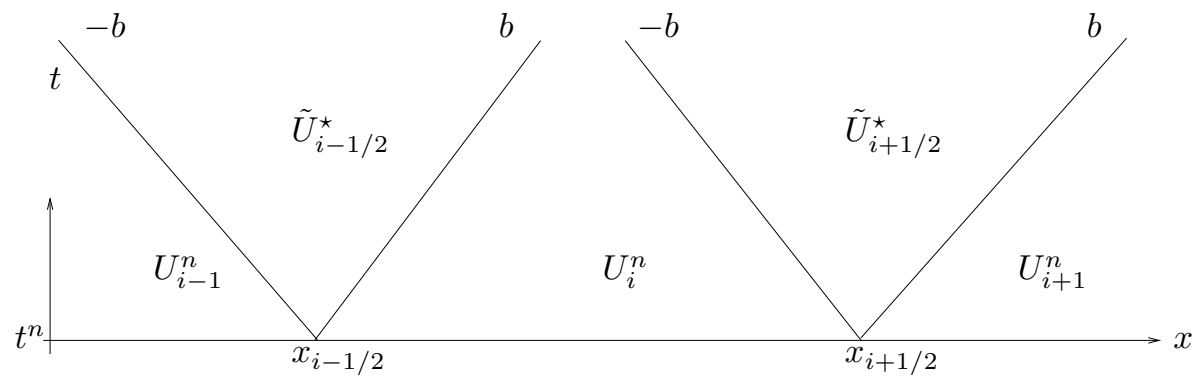

FiguRE 1. HLL scheme: Juxtaposition of approximated Riemann problems.

The updated approximated solution at time $t^{n+1}$ is thus defined as follows:

$$
\tilde{U}_{i}^{n+1}=\frac{1}{\Delta x} \int_{x_{i-1 / 2}}^{x_{i+1 / 2}} \tilde{U}_{\Delta x}^{n}\left(x, t^{n}+\Delta t\right) d x .
$$

Since we have

$$
\tilde{U}_{i+1 / 2}^{\star}=\frac{1}{2}\left(U_{i}^{n}+U_{i+1}^{n}\right)-\frac{1}{2 b}\left(F\left(U_{i+1}^{n}\right)-F\left(U_{i}^{n}\right)\right),
$$


an easy computation gives the following standard conservation form:

$$
\tilde{U}_{i}^{n+1}=U_{i}^{n}-\frac{\Delta t}{\Delta x}\left(F_{i+1 / 2}^{H L L}-F_{i-1 / 2}^{H L L}\right),
$$

where we have set

$$
F_{i+1 / 2}^{H L L}=\frac{1}{2}\left(F\left(U_{i}^{n}\right)+F\left(U_{i+1}^{n}\right)\right)-\frac{b}{2}\left(U_{i+1}^{n}-U_{i}^{n}\right) .
$$

For simplicity in the presentation, we have adopted here a constant numerical cone of dependence (cf. Figure 1) characterized by a single speed parameter $b>0$. As prescribed in 21. (cf. also [24, 32]), each cone of dependence can be variable and defined by a pair $\left(b_{i+1 / 2}^{-}, b_{i+1 / 2}^{+}\right)$with $b_{i+1 / 2}^{-}<b_{i+1 / 2}^{+}$. However, for the sake of simplicity and without genuine loss of generality, we present our strategy for the simpler case $b_{i+1 / 2}^{+}=-b_{i+1 / 2}^{-}=b>0$.

At this level, a remark must be made concerning the invariant domain property for the scheme (5.8). It suffices to ensure that $\tilde{U}_{i+1 / 2}^{\star}$ belongs to $\Omega$ for all $i \in \mathbb{Z}$ to deduce that $U_{i}^{n+1}$ in $\Omega$ for all $i \in \mathbb{Z}$. Indeed, from (5.6), we have

$$
\tilde{U}_{i}^{n+1}=b \frac{\Delta t}{\Delta x} \tilde{U}_{i-1 / 2}^{\star}+\left(1-2 b \frac{\Delta t}{\Delta x}\right) U_{i}^{n}+b \frac{\Delta t}{\Delta x} \tilde{U}_{i+1 / 2}^{\star}
$$

and, based on the CFL restriction (5.5), the above relation is a convex combination of states in $\Omega$. Since $\Omega$ is a convex set, we deduce that $\tilde{U}_{i}^{n+1}$ belongs to $\Omega$.

Our main necessary condition for the above argument is that $\tilde{U}_{i+1 / 2}^{\star} \in \Omega$ for all $i$ in $\mathbb{Z}$. But, once again, $\tilde{U}_{i+1 / 2}^{\star}$ can be seen as a convex combination, as follows:

$$
\tilde{U}_{i+1 / 2}^{\star}=\frac{1}{2}\left(U_{i}^{n}+\frac{1}{b} F\left(U_{i}^{n}\right)\right)+\frac{1}{2}\left(U_{i+1}^{n}-\frac{1}{b} F\left(U_{i+1}^{n}\right)\right) .
$$

Since $\Omega$ is an open convex set, we can choose $b$ to be large enough so as to enforce the condition $\tilde{U}_{i+1 / 2}^{\star} \in \Omega$.

Next, we modify this approximate Riemann solver and introduce a discretization of the source-term in order to approximate the solutions of (5.2). Similar modifications were made for specific problems in [3] and [2, 6], while we propose here a general approach based on matrix-valued free-parameters. We modify the approximate Riemann solver (5.3) as follows:

$$
U_{\mathcal{R}}\left(\frac{x}{t} ; U_{L}, U_{R}\right)= \begin{cases}U_{L}, & \frac{x}{t}<-b, \\ U^{\star L}, & -b<\frac{x}{t}<0, \\ U^{\star R}, & 0<\frac{x}{t}<b, \\ U_{R}, & \frac{x}{t}>b,\end{cases}
$$

where we have set

$$
\begin{aligned}
& U^{\star L}=\underline{\alpha} \tilde{U}^{\star}+(I-\underline{\alpha})\left(U_{L}-\bar{R}\left(U_{L}\right)\right), \\
& U^{\star R}=\underline{\alpha} \tilde{U}^{\star}+(I-\underline{\alpha})\left(U_{R}-\bar{R}\left(U_{R}\right)\right) .
\end{aligned}
$$

Here, $\underline{\alpha}$ denotes a $N \times N$ matrix defined as

$$
\underline{\alpha}=\left(I+\frac{\gamma \Delta x}{2 b}(I+\underline{\sigma})\right)^{-1},
$$


and

$$
\bar{R}(U)=(I+\underline{\sigma})^{-1} R(U) .
$$

The $N \times N$ matrices $I$ and $\underline{\sigma}$, respectively, denote the identity matrix and a parameter matrix to be defined.

A choice for the matrix $\underline{\sigma}$ will be made later, which will turn out to govern the asymptotic regime. At this point, the matrix $\underline{\sigma}$ is assumed to be such that the inverse matrices in (5.12) and (5.13) are well defined.

We adopt the modified approximate Riemann solver (5.10) to derive a modified Godunov type scheme in the spirit of [3]. At each cell interface $x_{i+1 / 2}$, we set the approximate Riemann solver $U_{\mathcal{R}}\left(\frac{x-x_{i+1 / 2}}{t-t^{n}} ; U_{i}^{n}, U_{i+1}^{n}\right)$ to define a juxtaposition of a modified approximate Riemann solver, denoted by $U_{\Delta x}^{n}\left(x, t^{n}+t\right)$ for $t \in[0, \Delta t)$. (See Figure 2)

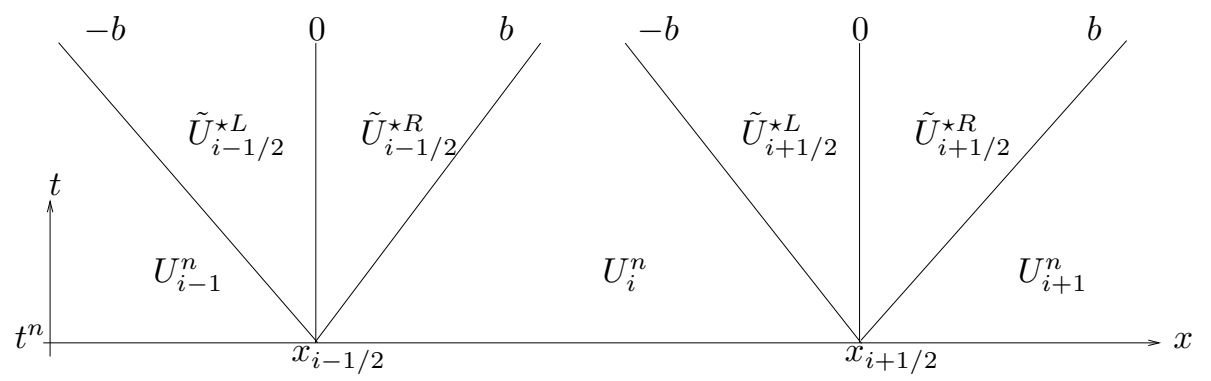

FiguRE 2. Juxtaposition of modified approximated Riemann problems.

Thanks to the CFL condition (5.5), such a juxtaposition is noninteracting.

At time $t^{n+1}$, the updated approximated solution is given as follows for all $i \in \mathbb{Z}$ :

$$
U_{i}^{n+1}=\int_{x_{i-1 / 2}}^{x_{i+1 / 2}} U_{\Delta x}^{n}\left(x, t^{n}+\Delta t\right) d x .
$$

We compute this integral form and obtain

$$
\begin{aligned}
& \frac{1}{\Delta t}\left(U_{i}^{n+1}-U_{i}^{n}\right)+\frac{1}{\Delta x}\left(\underline{\alpha}_{i+1 / 2} F_{i+1 / 2}^{H L L}-\underline{\alpha}_{i-1 / 2} F_{i-1 / 2}^{H L L}\right) \\
& =\frac{1}{\Delta x}\left(\underline{\alpha}_{i+1 / 2}-\underline{\alpha}_{i-1 / 2}\right) F\left(U_{i}^{n}\right)-\frac{b}{\Delta x}\left(I-\underline{\alpha}_{i-1 / 2}\right) \bar{R}_{i-1 / 2}\left(U_{i}^{n}\right) \\
& \quad-\frac{b}{\Delta x}\left(I-\underline{\alpha}_{i+1 / 2}\right) \bar{R}_{i+1 / 2}\left(U_{i}^{n}\right),
\end{aligned}
$$

where the numerical flux $F_{i+1 / 2}^{H L L}$ is given by (5.9). The discretized source-term can be rewritten in a more relevant form:

$$
\frac{b}{\Delta x}\left(I-\underline{\alpha}_{i+1 / 2}\right) \bar{R}_{i+1 / 2}\left(U_{i}^{n}\right)=\frac{b}{\Delta x} \underline{\alpha}_{i+1 / 2}\left(\underline{\alpha}_{i+1 / 2}^{-1}-I\right) \bar{R}_{i+1 / 2}\left(U_{i}^{n}\right) .
$$

In view of the definition of $\underline{\alpha}_{i+1 / 2}($ see $(\underline{5.12})$ ), we deduce that

and, similarly,

$$
\frac{b}{\Delta x}\left(I-\underline{\alpha}_{i+1 / 2}\right) \bar{R}_{i+1 / 2}\left(U_{i}^{n}\right)=\frac{\gamma}{2} \underline{\alpha}_{i+1 / 2} R\left(U_{i}^{n}\right)
$$

$$
\frac{b}{\Delta x}\left(I-\underline{\alpha}_{i-1 / 2}\right) \bar{R}_{i-1 / 2}\left(U_{i}^{n}\right)=\frac{\gamma}{2} \underline{\alpha}_{i-1 / 2} R\left(U_{i}^{n}\right) .
$$


As a consequence, the scheme (5.15) reads

$$
\begin{aligned}
& \frac{1}{\Delta t}\left(U_{i}^{n+1}-U_{i}^{n}\right)+\frac{1}{\Delta x}\left(\underline{\alpha}_{i+1 / 2} F_{i+1 / 2}^{H L L}-\underline{\alpha}_{i-1 / 2} F_{i-1 / 2}^{H L L}\right) \\
& =\frac{1}{\Delta x}\left(\underline{\alpha}_{i+1 / 2}-\underline{\alpha}_{i-1 / 2}\right) F\left(U_{i}^{n}\right)-\frac{\gamma}{2}\left(\underline{\alpha}_{i+1 / 2}+\underline{\alpha}_{i-1 / 2}\right) R\left(U_{i}^{n}\right) .
\end{aligned}
$$

This scheme satisfies the following statement.

Theorem 5.1. Assume that the matrix $\underline{\sigma}$ defines a spatially continuous map. The numerical scheme (5.16) is consistant with the equation (5.2).

At time $t^{n}$, assume $U_{i}^{n} \in \Omega$ for all $i \in \mathbb{Z}$ and, in addition, that the state vectors $U_{i+1 / 2}^{\star L}$ and $U_{i+1 / 2}^{\star R}$, defined by

$$
\begin{aligned}
& U_{i+1 / 2}^{\star L}=\underline{\alpha}_{i+1 / 2} \tilde{U}_{i+1 / 2}^{\star}+\left(I-\underline{\alpha}_{i+1 / 2}\right)\left(U_{i}^{n}-\bar{R}\left(U_{i}^{n}\right)\right), \\
& U_{i+1 / 2}^{\star R}=\underline{\alpha}_{i+1 / 2} \tilde{U}_{i+1 / 2}^{\star}+\left(I-\underline{\alpha}_{i+1 / 2}\right)\left(U_{i+1}^{n}-\bar{R}\left(U_{i+1}^{n}\right)\right),
\end{aligned}
$$

belong to $\Omega$. Then, the updated state vector $U_{i}^{n+1}$, defined by (5.16), belongs to the set $\Omega$ for all $i$ in $\mathbb{Z}$.

Proof. The consistency property follows from the definition of $\underline{\alpha}_{i+1 / 2}$, given by (5.12). Indeed, we easily obtain

$$
\underline{\alpha}_{i+1 / 2}=I+\mathcal{O}(\Delta x),
$$

to deduce the expected consistency of the flux and the relaxation source term. Only the term $\frac{1}{\Delta x}\left(\underline{\alpha}_{i+1 / 2}-\underline{\alpha}_{i-1 / 2}\right) F\left(U_{i}^{n}\right)$ remains. Recalling (5.12), we have

$$
\frac{1}{\Delta x}\left(\underline{\alpha}_{i+1 / 2}-\underline{\alpha}_{i-1 / 2}\right) F\left(U_{i}^{n}\right)=-\frac{\gamma}{2 b} \underline{\alpha}_{i+1 / 2}\left(\underline{\sigma}_{i+1 / 2}-\underline{\sigma}_{i-1 / 2}\right) \underline{\alpha}_{i-1 / 2} F\left(U_{i}^{n}\right),
$$

to obtain

$$
\frac{1}{\Delta x}\left(\underline{\alpha}_{i+1 / 2}-\underline{\alpha}_{i-1 / 2}\right) F\left(U_{i}^{n}\right)=\mathcal{O}(\Delta x),
$$

as soon as $\underline{\sigma}_{i+1 / 2}-\underline{\sigma}_{i-1 / 2}=\mathcal{O}(\Delta x)$. The expected equation consistency is therefore obtained.

Concerning the robustness of the method, from (5.14), we have

$$
U_{i}^{n+1}=b \frac{\Delta t}{\Delta x} U_{i-1 / 2}^{\star L}+\left(1-2 b \frac{\Delta t}{\Delta x}\right) U_{i}^{n}+b \frac{\Delta t}{\Delta x} U_{i+1 / 2}^{\star R},
$$

which is nothing but a convex combination in $\Omega$. Then $U_{i}^{n+1}$ is in $\Omega$ and the proof is completed.

To conclude this derivation, observe that the term $\frac{1}{\Delta x}\left(\underline{\alpha}_{i+1 / 2}-\underline{\alpha}_{i-1 / 2}\right) F\left(U_{i}^{n}\right)$ may seem to be a discrepancy in the method. In fact, this term is standard to derive asymptotic preserving schemes and it can be found in several works. (See, for instance, [2, 3, 6].) Our approach allows us to recover a scheme proposed earlier in 18 . 
The linear asymptotic regime. The scheme (5.16) is now considered to suggest a discretization of our initial model (1.1). The expected scheme is thus easily obtained when substituting $\Delta t$ by $\frac{\Delta t}{\varepsilon}$ and fixing $\gamma=\frac{1}{\varepsilon}$. The resulting scheme reads as follows:

$$
\begin{aligned}
& \frac{\varepsilon}{\Delta t}\left(U_{i}^{n+1}-U_{i}^{n}\right)+\frac{1}{\Delta x}\left(\underline{\alpha}_{i+1 / 2} F_{i+1 / 2}^{H L L}-\underline{\alpha}_{i-1 / 2} F_{i-1 / 2}^{H L L}\right) \\
& =\frac{1}{\Delta x}\left(\underline{\alpha}_{i+1 / 2}-\underline{\alpha}_{i-1 / 2}\right) F\left(U_{i}^{n}\right)-\frac{1}{2 \varepsilon}\left(\underline{\alpha}_{i+1 / 2}+\underline{\alpha}_{i-1 / 2}\right) R\left(U_{i}^{n}\right),
\end{aligned}
$$

where

$$
\underline{\alpha}_{i+1 / 2}=\left(I+\frac{\Delta x}{2 \varepsilon b}\left(I+\underline{\sigma}_{i+1 / 2}\right)\right)^{-1} .
$$

For the sake of simplicity in the forthcoming asymptotic derivation, we propose to introduce the $N \times N$ matrix $\underline{\alpha}_{i+1 / 2}^{\varepsilon}$ defined by

$$
\underline{\alpha}_{i+1 / 2}^{\varepsilon}=\left(\varepsilon I+\frac{\Delta x}{2 b}\left(I+\underline{\sigma}_{i+1 / 2}\right)\right)^{-1},
$$

so that we have $\underline{\alpha}_{i+1 / 2}=\varepsilon \underline{\alpha}_{i+1 / 2}^{\varepsilon}$. Recalling the definition, the scheme (5.16) takes the form:

$$
\begin{aligned}
& \frac{\varepsilon}{\Delta t}\left(U_{i}^{n+1}-U_{i}^{n}\right)+\frac{\varepsilon}{\Delta x}\left(\underline{\alpha}_{i+1 / 2}^{\varepsilon} F_{i+1 / 2}^{H L L}-\underline{\alpha}_{i-1 / 2}^{\varepsilon} F_{i-1 / 2}^{H L L}\right) \\
& =\frac{\varepsilon}{\Delta x}\left(\underline{\alpha}_{i+1 / 2}^{\varepsilon}-\underline{\alpha}_{i-1 / 2}^{\varepsilon}\right) F\left(U_{i}^{n}\right)-\frac{1}{2}\left(\underline{\alpha}_{i+1 / 2}^{\varepsilon}+\underline{\alpha}_{i-1 / 2}^{\varepsilon}\right) R\left(U_{i}^{n}\right) .
\end{aligned}
$$

We observe that $U_{i}^{n}$ remains close to the equilibrium state $\mathcal{E}\left(u_{i}^{n}\right)$ for $\varepsilon$ small and we thus consider the following expansion:

$$
U_{i}^{n}=\mathcal{E}\left(u_{i}^{n}\right)+\varepsilon\left(U_{1}\right)_{i}^{n}+\mathcal{O}\left(\varepsilon^{2}\right),
$$

which we now plug into (5.20). We easily have

$$
\begin{aligned}
& \frac{1}{\varepsilon} R\left(U_{i}^{n}\right)=B\left(\mathcal{E}\left(u_{i}^{n}\right)\right) \cdot\left(U_{1}\right)_{i}^{n}+\mathcal{O}(\varepsilon), \\
& \left.F_{i+1 / 2}^{H L L}\right|_{\mathcal{E}(u)+\mathcal{O}(\varepsilon)}=\left.F_{i+1 / 2}^{H L L}\right|_{\mathcal{E}(u)}+\mathcal{O}(\varepsilon),
\end{aligned}
$$

where

$$
\left.F_{i+1 / 2}^{H L L}\right|_{\mathcal{E}(u)}=\frac{1}{2}\left(F\left(\mathcal{E}\left(u_{i}^{n}\right)\right)+F\left(\mathcal{E}\left(u_{i+1}^{n}\right)\right)\right)-\frac{b}{2}\left(\mathcal{E}\left(u_{i+1}^{n}\right)-\mathcal{E}\left(u_{i}^{n}\right)\right) .
$$

In addition we have

$$
\underline{\alpha}_{i+1 / 2}^{\varepsilon}=\frac{2 b}{\Delta x}\left(I+\underline{\sigma}_{i+1 / 2}\right)^{-1}+\mathcal{O}(\varepsilon) .
$$

By considering the first-order terms resulting from (5.20), we obtain

$$
\begin{aligned}
& \frac{1}{\Delta t}\left(\mathcal{E}\left(u_{i}^{n+1}\right)-\mathcal{E}\left(u_{i}^{n}\right)\right) \\
& +\frac{2 b}{\Delta x^{2}}\left(\left.\left(I+\underline{\sigma}_{i+1 / 2}\right)^{-1} F_{i+1 / 2}^{H L L}\right|_{\mathcal{E}(u)}-\left.\left(I+\underline{\sigma}_{i-1 / 2}\right)^{-1} F_{i-1 / 2}^{H L L}\right|_{\mathcal{E}(u)}\right) \\
& =\frac{2 b}{\Delta x^{2}}\left(\left(I+\underline{\sigma}_{i+1 / 2}\right)^{-1}-\left(I+\underline{\sigma}_{i-1 / 2}\right)^{-1}\right) F\left(\mathcal{E}\left(u_{i}^{n}\right)\right) \\
& \quad-\frac{b}{\Delta x}\left(\left(I+\underline{\sigma}_{i+1 / 2}\right)^{-1}+\left(I+\underline{\sigma}_{i-1 / 2}\right)^{-1}\right) B\left(\mathcal{E}\left(u_{i}^{n}\right)\right) \cdot\left(U_{1}\right)_{i}^{n} .
\end{aligned}
$$


Next, assume the existence of an $n \times n$ squared matrix, denoted by $\mathcal{M}_{i+1 / 2}$, such that

$$
Q\left(I+\underline{\sigma}_{i+1 / 2}\right)^{-1}=\frac{1}{b^{2}} \mathcal{M}_{i+1 / 2} Q .
$$

Recalling the assumptions (1.2), (1.4), and (1.7), we write

$$
\frac{1}{\Delta t}\left(u_{i}^{n+1}-u_{i}^{n}\right)=-\frac{2}{b \Delta x^{2}}\left(\left.\mathcal{M}_{i+1 / 2} Q F_{i+1 / 2}^{H L L}\right|_{\mathcal{E}(u)}-\left.\mathcal{M}_{i-1 / 2} Q F_{i-1 / 2}^{H L L}\right|_{\mathcal{E}(u)}\right),
$$

where

$$
\begin{aligned}
\left.Q F_{i+1 / 2}^{H L L}\right|_{\mathcal{E}(u)} & =\frac{1}{2} Q\left(F\left(\mathcal{E}\left(u_{i}^{n}\right)\right)+F\left(\mathcal{E}\left(u_{i+1}^{n}\right)\right)\right)-\frac{b}{2} Q\left(\mathcal{E}\left(u_{i+1}^{n}\right)-\mathcal{E}\left(u_{i}^{n}\right)\right) \\
& =-\frac{b}{2}\left(u_{i+1}^{n}-u_{i}^{n}\right) .
\end{aligned}
$$

As a consequence, the asymptotic discrete regime is given by

$$
\frac{1}{\Delta t}\left(u_{i}^{n+1}-u_{i}^{n}\right)=\frac{1}{\Delta x^{2}}\left(\mathcal{M}_{i+1 / 2}\left(u_{i+1}^{n}-u_{i}^{n}\right)+\mathcal{M}_{i-1 / 2}\left(u_{i-1}^{n}-u_{i}^{n}\right)\right) .
$$

Thus, we have established the following result.

Theorem 5.2. Consider any $N \times N$ matrix $\underline{\sigma}_{i+1 / 2}$ such that the matrices $I+\underline{\sigma}_{i+1 / 2}$ and $\left(1+\frac{\Delta x}{2 \varepsilon b}\right) I+\underline{\sigma}_{i+1 / 2}$ are nonsingular for all $\varepsilon>0$. Assume the existence of an $n \times n$ matrix $\mathcal{M}_{i+1 / 2}$ such that (5.21) holds, and introduce the $n \times n$ matrix $\mathcal{M}(u)$ defined by

$$
\mathcal{M}(u)=Q A(\mathcal{E}(u)) \mathcal{L}^{-1}(u) D_{U}^{2} \Phi(\mathcal{E}(u)) A(\mathcal{E}(u)) D_{u} \mathcal{E}(u) .
$$

In addition, assume that the matrix $\mathcal{M}_{i+1 / 2}$ is a discrete form of $\mathcal{M}(u)$ at each cell interface $x_{i+1 / 2}$. Then, the asymptotic behavior of the scheme (5.20) coincides with a discrete form for the limit diffusion equation (2.13).

Proof. We directly deduce from (2.13) that the diffusive limit equation reads

$$
\partial_{t} u=\partial_{x}\left(\mathcal{M}(u) \partial_{x} u\right) .
$$

Since the asymptotic regime satisfied by the scheme (5.20) is governed by (5.22), the proposed choice of the matrix $\mathcal{M}_{i+1 / 2}$ leads us to the correct behavior of the scheme as $\varepsilon$ goes to zero.

The nonlinear asymptotic regime. We propose to extend the above numerical scheme to consider the nonlinear asymptotic regime governed by the system (3.1). To address such an issue, once again we consider the scheme (5.16) where $\Delta t$ is substituted by $\frac{\Delta t}{\varepsilon}$ and we set $\gamma=\frac{1}{\varepsilon}$. To be consistent, we substitute $R\left(U_{i}^{n}\right)$ by $\frac{1}{\varepsilon^{m-1}} R\left(U_{i}^{n}\right)$.

Adopting such a strategy, the same arguments used to obtain (5.20) now give

$$
\begin{aligned}
& \frac{1}{\Delta t}\left(U_{i}^{n+1}-U_{i}^{n}\right)+\frac{1}{\Delta x}\left(\underline{\alpha}_{i+1 / 2}^{\varepsilon} F_{i+1 / 2}^{H L L}-\underline{\alpha}_{i-1 / 2}^{\varepsilon} F_{i-1 / 2}^{H L L}\right) \\
& =\frac{1}{\Delta x}\left(\underline{\alpha}_{i+1 / 2}^{\varepsilon}-\underline{\alpha}_{i-1 / 2}^{\varepsilon}\right) F\left(U_{i}^{n}\right)-\frac{1}{2 \varepsilon^{m}}\left(\underline{\alpha}_{i+1 / 2}^{\varepsilon}+\underline{\alpha}_{i-1 / 2}^{\varepsilon}\right) R\left(U_{i}^{n}\right),
\end{aligned}
$$

where $\underline{\alpha}_{i+1 / 2}^{\varepsilon}$ is defined by (5.19). The above scheme exactly coincides with (5.20) as soon as we fix $m=1$. 
Once again, as soon as $\varepsilon$ tends to zero, the state vector $U_{i}^{n}$ remains in a neighborhood of the equilibrium state $\mathcal{E}\left(u_{i}^{n}\right)$. As a consequence, we adopt a formal expansion given by

$$
U_{i}^{n}=\mathcal{E}\left(u_{i}^{n}\right)+\varepsilon\left(U_{1}\right)_{i}^{n}+\mathcal{O}\left(\varepsilon^{2}\right) .
$$

Arguing the same calculations as used in the linear expansion case, the asymptotic discrete equation is given by (5.22). Hence, we have to choose $\mathcal{M}_{i+1 / 2}$ in order to get a discretization of (3.6).

Theorem 5.3. Consider an $N \times N$ matrix $\underline{\sigma}_{i+1 / 2}$ such that the matrices $I+\underline{\sigma}_{i+1 / 2}$ and $\left(1+\frac{\Delta x}{2 \varepsilon b}\right) I+\underline{\sigma}_{i+1 / 2}$ are nonsingular for all $\varepsilon>0$. Assume the existence of an $n \times n$ matrix $\mathcal{M}_{i+1 / 2}$ such that (5.21) holds. Consider also the $n \times n$ matrix $\mathcal{M}(u)$ defined by

$$
\mathcal{M}(u)=Q A(\mathcal{E}(u)) \mathcal{R}^{-1}(u),
$$

where $\mathcal{R}^{-1}: \omega \rightarrow \Omega$ defines the unique solution of (3.5). Assume that $\mathcal{M}_{i+1 / 2}$ is a discrete form of $\mathcal{M}(u)$ at each cell interface $x_{i+1 / 2}$. The asymptotic behavior of the scheme (5.23) defines a discrete form of the nonlinear diffusion equation (3.6).

Proof. By definition of $\mathcal{R}^{-1}(u)$, we have $\bar{U}_{1}=\mathcal{R}^{-1}(u)$, the unique solution to (3.5). As a consequence, we deduce the following rewriting of (3.6):

$$
\partial_{t} u=\partial_{x}\left(\mathcal{M}(u) \partial_{x} u\right),
$$

where $\mathcal{M}(u)$ is given by (5.24). The proposed definition of $\mathcal{M}_{i+1 / 2}$ concludes the proof.

\section{Numerical EXPERIMENTS}

Euler equations with friction. To illustrate the interest of the asymptotic preserving scheme (5.17), we now apply it to the Euler equations with the friction term (4.1). Here, we suggest to fix the matrix parameter $\underline{\sigma}_{i+1 / 2}$ as follows:

$$
\underline{\sigma}_{i+1 / 2}=\sigma_{i+1 / 2} I
$$

where $\sigma_{i+1 / 2}$ stands for a scalar parameter to be defined. As a consequence, the matrix $\underline{\alpha}_{i+1 / 2}$, defined by (5.18), is now given by

$$
\underline{\alpha}_{i+1 / 2}=\alpha_{i+1 / 2} I, \quad \alpha_{i+1 / 2}=\frac{1}{1+\frac{\Delta x}{2 \varepsilon b}\left(1+\sigma_{i+1 / 2}\right)} .
$$

The scheme (5.17) thus reads

$$
\begin{aligned}
& \frac{\varepsilon}{\Delta t}\left(\rho_{i}^{n+1}-\rho_{i}^{n}\right)+\frac{1}{\Delta x}\left(\alpha_{i+1 / 2} F_{i+1 / 2}^{\rho, H L L}-\alpha_{i-1 / 2} F_{i-1 / 2}^{\rho, H L L}\right) \\
& =-\alpha_{i+1 / 2} \frac{\sigma_{i+1 / 2}-\sigma_{i-1 / 2}}{2 \varepsilon b} \alpha_{i-1 / 2}(\rho v)_{i}^{n}, \\
& \frac{\varepsilon}{\Delta t}\left((\rho v)_{i}^{n+1}-(\rho v)_{i}^{n}\right)+\frac{1}{\Delta x}\left(\alpha_{i+1 / 2} F_{i+1 / 2}^{\rho v, H L L}-\alpha_{i-1 / 2} F_{i-1 / 2}^{\rho v, H L L}\right) \\
& =-\alpha_{i+1 / 2} \frac{\sigma_{i+1 / 2}-\sigma_{i-1 / 2}}{2 \varepsilon b} \alpha_{i-1 / 2}\left(\rho_{i}^{n}\left(v_{i}^{n}\right)^{2}+p\left(\rho_{i}^{n}\right)\right) \\
& -\frac{1}{\varepsilon} \frac{\alpha_{i+1 / 2}+\alpha_{i-1 / 2}}{2}(\rho v)_{i}^{n},
\end{aligned}
$$

where the numerical flux vector $\left(F_{i+1 / 2}^{\rho, H L L}, F_{i+1 / 2}^{\rho v, H L L}\right)$ is defined by (5.9). 
First, applying Theorem [5.1, we observe that the proposed scheme is consistent with the system (4.1) and preserves the admissible states. To address such an issue, in view of Theorem 5.1, we have to establish the positivity property $(i \in \mathbb{Z})$ as follows:

$$
\begin{aligned}
& \rho_{i+1 / 2}^{\star L}=\alpha_{i+1 / 2} \tilde{\rho}_{i+1 / 2}^{\star}+\left(1-\alpha_{i+1 / 2}\right) \rho_{i}^{n}, \\
& \rho_{i+1 / 2}^{\star R}=\alpha_{i+1 / 2} \tilde{\rho}_{i+1 / 2}^{\star}+\left(1-\alpha_{i+1 / 2}\right) \rho_{i+1}^{n} .
\end{aligned}
$$

Since $\alpha_{i+1 / 2}$, defined by (6.1), belongs to $(0,1)$, we have $\rho_{i+1 / 2}^{\star L}>0$ and $\rho_{i+1 / 2}^{\star R}>0$ as soon as $\tilde{\rho}_{i+1 / 2}^{\star}>0$, which is satisfied for sufficiently large values of $b$ (cf. relation (5.4) ).

Now, we study for the asymptotic behavior of the scheme (6.2)-(6.3). In other words, we have to fix the free parameter $\sigma_{i+1 / 2}$ to recover the expected asymptotic regime in the limit of $\varepsilon$ to zero. This required asymptotic behavior must be governed by a discrete form of (4.3). First, observe that

$$
\lim _{\varepsilon \rightarrow 0} \alpha_{i+1 / 2}=0, \quad \lim _{\varepsilon \rightarrow 0} \frac{\alpha_{i+1 / 2}}{\varepsilon}=0
$$

From the momentum $(\rho v)_{i}^{n+1}$ governing equation (6.2), we easily deduce the following momentum behavior in the limit of $\varepsilon$ to zero:

$$
(\rho v)_{i}^{n}=0, \quad i \in \mathbb{Z} .
$$

The density approximation thus admits the following asymptotic regime:

$$
\frac{1}{\Delta t}\left(\rho_{i}^{n+1}-\rho_{i}^{n}\right)+\frac{2 b}{\delta x^{2}}\left(\left.\frac{1}{1+\sigma_{i+1 / 2}} F_{i+1 / 2}^{\rho, H L L}\right|_{\rho v=0}-\left.\frac{1}{1+\sigma_{i-1 / 2}} F_{i-1 / 2}^{\rho, H L L}\right|_{\rho v=0}\right)=0 .
$$

But we have

and therefore,

$$
\left.F_{i-1 / 2}^{\rho, H L L}\right|_{\rho v=0}=-\frac{b}{2}\left(\rho_{i+1}^{n}-\rho_{i}^{n}\right)
$$

$$
\frac{1}{\Delta t}\left(\rho_{i}^{n+1}-\rho_{i}^{n}\right)=\frac{b^{2}}{\delta x^{2}}\left(\frac{1}{1+\sigma_{i+1 / 2}}\left(\rho_{i+1}^{n}-\rho_{i}^{n}\right)+\frac{1}{1+\sigma_{i-1 / 2}}\left(\rho_{i-1}^{n}-\rho_{i}^{n}\right)\right) .
$$

We choose

$$
\sigma_{i+1 / 2}=\left\{\begin{array}{l}
b^{2} \frac{\rho_{i+1}^{n}-\rho_{i}^{n}}{p\left(\rho_{i+1}^{n}\right)-p\left(\rho_{i}^{n}\right)}-1, \quad \text { if } \rho_{i+1}^{n} \neq \rho_{i}^{n}, \\
\frac{b^{2}}{p^{\prime}\left(\rho_{i}^{n}\right)}-1, \quad \text { otherwise, }
\end{array}\right.
$$

and arrive at the following discretization of the diffusion equation (4.3):

$$
\frac{1}{\Delta t}\left(\rho_{i}^{n+1}-\rho_{i}^{n}\right)=\frac{1}{\delta x^{2}}\left(p\left(\rho_{i+1}^{n}\right)-2 p\left(\rho_{i}^{n}\right)+p\left(\rho_{i-1}^{n}\right)\right) .
$$

To conclude, observe that Theorem 5.2 applies if the matrix $\mathcal{M}_{i+1 / 2}$ is defined by $\mathcal{M}_{i+1 / 2}=m_{i+1 / 2} I_{n}$ where $I_{n}$ denotes the $n \times n$ identity matrix and

$$
m_{i+1 / 2}=\left\{\begin{array}{l}
\frac{p\left(\rho_{i+1}^{n}\right)-p\left(\rho_{i}^{n}\right)}{\rho_{i+1}^{n}-\rho_{i}^{n}}, \quad \text { if } \rho_{i+1}^{n} \neq \rho_{i}^{n}, \\
p^{\prime}\left(\rho_{i}^{n}\right), \quad \text { otherwise. }
\end{array}\right.
$$

In [3, 9, the scheme (6.2) 6.3 was derived by a completely different approach. Similarly, in [3, 2, 6], the application of our general asymptotic preserving scheme (5.17) is found in the framework of the radiative transfer (4.4). 


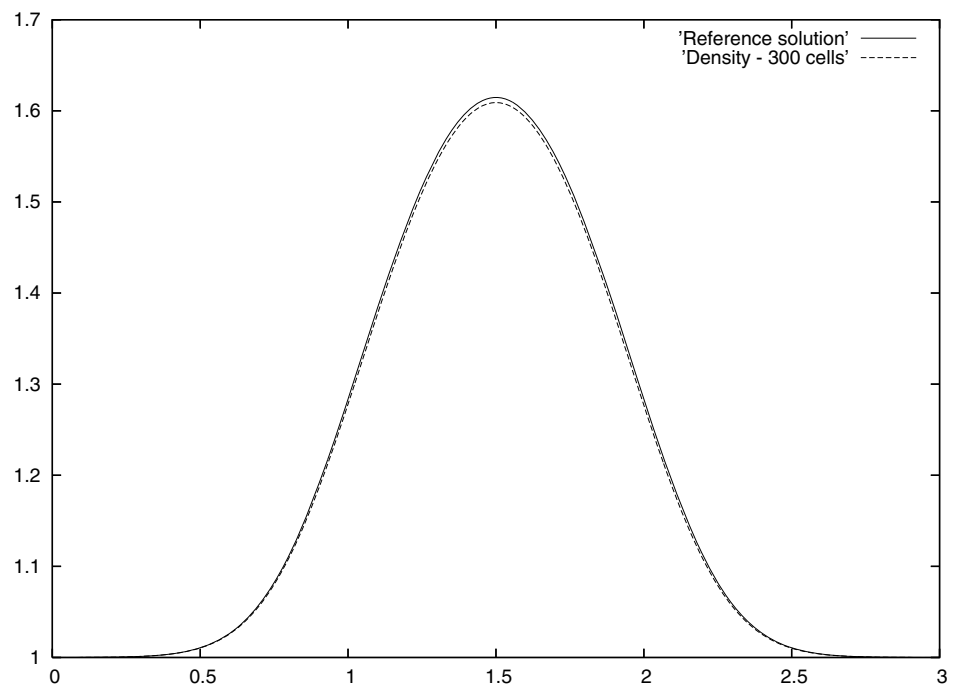

Figure 3. Reference (full line) and proposed scheme (dashed line) solution comparison.

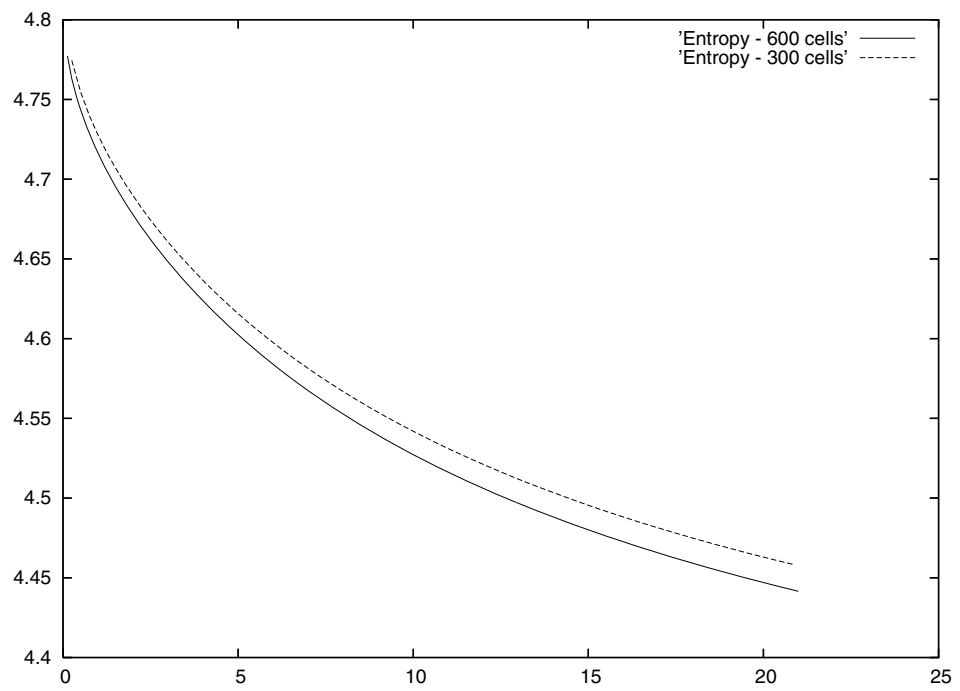

FiguRE 4. Decrease of the entropy.

As an illustration, the scheme (6.2) - (6.3) supplemented by the asymptotic preserving correction (6.4), is used to approximate the solution when the initial data is given by

$$
(\rho, \rho v)^{T}= \begin{cases}(2,0)^{T}, & x \in[1.2,1.8] \\ (1,0)^{T}, & \text { otherwise }\end{cases}
$$

Furthermore, we choose the simple pressure law $p(\rho)=\rho^{2}$ and $\varepsilon=10^{-3}$. In Figure 3, the solution on a 300 cells grid $\left(\Delta x=10^{-2}\right)$ is compared at time $t=$ 
$2.10^{4}$ with a numerical approximation of (4.3) computed with 600 cells. We note fairly good agreement between the two approximate results. In agreement with Proposition 2.3. Figure 4 shows that entropy is decreasing in time. For two choices of space grids, we plot $\sum_{i}\left(\rho \frac{v^{2}}{2}+\rho e(\rho)\right)_{i}$ versus time.

\section{Density}
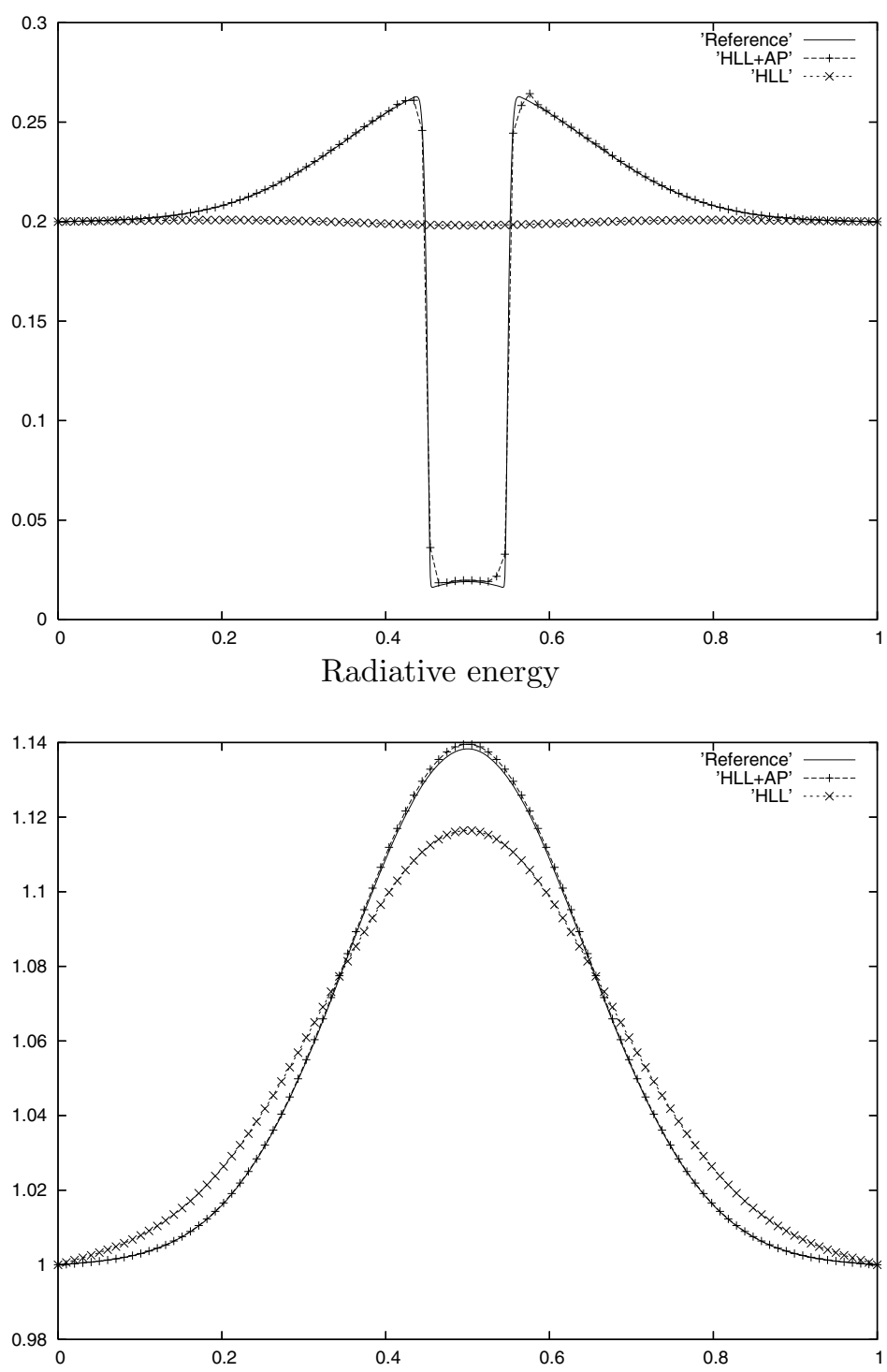

FiguRE 5. Reference (full line) and proposed scheme (dashed lines) solution with and without asymptotic preserving correction. Density $\rho$ is shown above and Radiative energy $e$ is shown below. 
Coupled Euler/M1 equations. We now propose to approximate the solution of the system (4.5) by adopting the asymptotic-preserving scheme (5.17) with the following matrix parameter $\underline{\sigma}_{i+1 / 2}$ :

$$
\underline{\sigma}_{i+1 / 2}=\left(\begin{array}{cccc}
\underline{\sigma}_{1, i+1 / 2} & 0 & -\underline{\sigma}_{2, i+1 / 2} & 0 \\
0 & 0 & 0 & 0 \\
0 & 0 & \underline{\sigma}_{3, i+1 / 2} & 0 \\
0 & 0 & 0 & 0
\end{array}\right),
$$

where $\underline{\sigma}_{j, i+1 / 2}$ are parameters to be defined later, in order to reach the required asymptotic regime (4.6). Such a definition $\underline{\alpha}_{i+1 / 2}^{\varepsilon}$, defined by (5.19), becomes:

$$
\underline{\alpha}_{k}^{\varepsilon}=\left(\begin{array}{cccc}
\frac{2 b_{k} \varepsilon}{\theta_{1, k}} & 0 & \frac{2 b_{k} \varepsilon \gamma \Delta x \underline{\sigma}_{2, k}}{\theta_{1, k} \theta_{3, k}} & 0 \\
0 & \frac{2 b_{k} \varepsilon}{2 b_{k} \varepsilon+\gamma \Delta x} & 0 & 0 \\
0 & 0 & \frac{2 b_{k} \varepsilon}{\theta_{3, k}} & 0 \\
0 & 0 & 0 & \frac{2 b_{k} \varepsilon}{2 b_{k} \varepsilon+\gamma \Delta x}
\end{array}\right),
$$

where $\theta_{j, k}=2 b_{k} \varepsilon+\gamma \Delta x\left(1+\underline{\sigma}_{j, k}\right)$ and $\gamma=\frac{\max (\kappa, \sigma)}{\varepsilon}$. The asymptotic regime associated with this scheme is

$$
\begin{aligned}
\rho_{i}^{n+1}= & \rho_{i}^{n}-\frac{\Delta t}{\Delta x} \frac{b^{2}}{\gamma \Delta x}\left(\frac{\rho_{i+1}^{n}-\rho_{i}^{n}}{1+\underline{\sigma}_{1, i+1 / 2}}-\frac{\rho_{i}^{n}-\rho_{i-1}^{n}}{1+\underline{\sigma}_{1, i-1 / 2}}\right. \\
& \left.+\frac{\underline{\sigma}_{2, i+1 / 2}\left(e_{i+1}^{n}-e_{i}^{n}\right)}{\left(1+\underline{\sigma}_{1, i+1 / 2}\right)\left(1+\underline{\sigma}_{3, i+1 / 2}\right)}-\frac{\underline{\sigma}_{2, i-1 / 2}\left(e_{i}^{n}-e_{i-1}^{n}\right)}{\left(1+\underline{\sigma}_{1, i-1 / 2}\right)\left(1+\underline{\sigma}_{3, i-1 / 2}\right)}\right), \\
e_{i}^{n+1}= & e_{i}^{n}-\frac{\Delta t}{\Delta x} \frac{b^{2}}{\gamma \Delta x}\left(\frac{e_{i+1}^{n}-e_{i}^{n}}{1+\underline{\sigma}_{3, i+1 / 2}}-\frac{e_{i}^{n}-e_{i-1}^{n}}{1+\underline{\sigma}_{3, i-1 / 2}}\right) .
\end{aligned}
$$

To recover a discrete form of (4.6), we propose to set

$$
\begin{aligned}
\underline{\sigma}_{1, i+1 / 2} & =\frac{\kappa}{\gamma} \frac{\rho_{i+1}^{n}-\rho_{i}^{n}}{p_{i+1}^{n}-p_{i}^{n}}-1, \\
\underline{\sigma}_{2, i+1 / 2} & =\frac{\sigma}{\gamma} \frac{\rho_{i+1}^{n}-\rho_{i}^{n}}{p_{i+1}^{n}-p_{i}^{n}}, \\
\underline{\sigma}_{3, i+1 / 2} & =\frac{3 \sigma}{\gamma}-1 .
\end{aligned}
$$

In conclusion, setting $\Delta p_{i+1 / 2}^{n}=\frac{p_{i+1}^{n}-p_{i}^{n}}{\rho_{i+1}^{n}-\rho_{i}^{n}}$ we obtain

$$
\underline{\sigma}_{i+1 / 2}=\left(\begin{array}{cccc}
\frac{\kappa}{\gamma \Delta p_{i+1 / 2}^{n}}-1 & 0 & -\frac{\sigma}{\gamma \Delta p_{i+1 / 2}^{n}} & 0 \\
0 & 0 & 0 & 0 \\
0 & 0 & \frac{3 \sigma}{\gamma}-1 & 0 \\
0 & 0 & 0 & 0
\end{array}\right) \text {, }
$$


This scheme is now applied to the following numerical experiment. We choose initial data given by

$$
\rho=0.2, \quad v=0, \quad f=0, \quad e= \begin{cases}1.5, & x \in[0.45,0.55] \\ 1, & \text { otherwise }\end{cases}
$$

The parameters of the model are $\kappa=2, \sigma=1, \varepsilon=10^{-3}, C_{p}=10^{-3}$ and $\eta=2$. The numerical solution is computed with $\Delta x=10^{-2}$ and compared to Figure 5 with a reference solution obtained by solving (4.6). Once again, the solution is perfectly captured, even on a coarse grid. We emphasize that this case is very challenging because of the very specific form of diffusion involved on the density.

\section{Concluding REMARKS}

We have presented a general framerwork to investigate the late-time/stiff-relaxation limit of a large class of hyperbolic systems. This framework was shown to cover the examples of interest arising in the modeling of complex fluid flows when several time-scales are involved. A new class of schemes was proposed for their approximation, and we demonstrated that the proposed modification was crucial in order to ensure the correct asymptotic behavior for late times. It would be interesting to make comparisons between the numerical results obtained here and concrete experimental results, especially on radiative transfer problems. In future work, we plan to generalize our continuous and discrete frameworks to problems with several space dimensions, and to numerically investigate the robustness and accuracy of such multi-dimensional finite volume discretizations in realistic physical situations.

\section{ACKNOWLEDGMENTS}

The authors gratefully thank Eric Paturel and Friedrich Wagemann for fruitful discussions. The second author (PLF) was partially supported by the Agence Nationale de la Recherche (ANR) through the grant 06-2-134423, and by the Centre National de la Recherche Scientifique (CNRS).

\section{REFERENCES}

[1] D. Aregba-Driollet, R. Natalini, Convergence of relaxation schemes for conservation laws, Appl. Anal., 61, no. 1-2, 163-193 (1996). MR1625520

[2] C. Berthon, P. Charrier, B. Dubroca, An HLLC scheme to solve the M1 Model of radiative transfer in two space dimensions, J. Sci. Comput., 31, no. 3, 347-389 (2007). MR.2320554 (2008f:85004)

[3] C. Berthon, R. Turpault, Asymptotic preserving HLL schemes, Numer. Methods Partial Differential Equations, DOI: 10.1002/num.20586.

[4] S. Bianchini, B. Hanouzet, R. Natalini, Asymptotic behavior of smooth solutions for partially dissipative hyperbolic systems with a convex entropy, Comm. Pure Appl. Math., 60, 15591622 (2007). MR2349349 (2010i:35227)

[5] F. Bouchut, H. Ounaissa, B. Perthame, Upwinding of the source term at interfaces for Euler equations with high friction, J. Comput. Math. Appl. 53, No. 3-4, 361-375 (2007). MR.2323698(2008e:76120)

[6] C. Buet, S. Cordier, An asymptotic preserving scheme for hydrodynamics radiative transfer models: numerics for radiative transfer, Numer. Math. 108, 199-221 (2007). MR2358003 (2008i:76138)

[7] C. Buet, B. Després, Asymptotic preserving and positive schemes for radiation hydrodynamics, J. Comput. Phys. 215, 717-740 (2006). MR2219880 (2007j:85005) 
[8] C. Cercignani, The Boltzmann equation and its applications, Applied Math. Sciences, Vol. 67, Springer-Verlag, New York, 1988. MR1313028 (95i:82082)

[9] C. Chalons, F. Coquel, E. Godlewski, P.-A. Raviart, N. Seguin Godunov-type schemes for hyperbolic systems with parameter dependent source. The case of Euler system with friction, Math. Model. Methods Appl. Sci. 20 (2010), no. 11, 2109-2166. MR2740716 (2011m:65179)

[10] C. Chalons, J.-F. Coulombel, Relaxation approximation of the Euler equations, J. Math. Anal. Appl., vol 348, no. 2, 872-893 (2008). MR2446042 (2010b:35349)

[11] G.Q. Chen, C.D. Levermore, T.P. Liu, Hyperbolic conservation laws with stiff-relaxation terms and entropy, Comm. Pure Appl. Math. 47, 787-830 (1995). MR.1280989 (95h:35133)

[12] F. Coquel, B. Perthame, Relaxation of energy and approximate Riemann solvers for general pressure laws in fluid dynamics, SIAM J. Numer. Anal. 35, 2223-2249 (1998). MR.1655844 (2000a:76129)

[13] P. Crispel, P. Degond, M.-H. Vignal, An asymptotic preserving scheme for the two-fluid EulerPoisson model in the quasi-neutral limit, J. Comput. Phys. 223, 208-234 (2007). MR2314389 (2008b:76205)

[14] P. Degond, F. Deluzet, A. Sangam, M.-H. Vignal, An asymptotic preserving scheme for the Euler equations in a strong magnetic field, J. Comput. Phys. 228, 3540-3558 (2009). MR.2511066 (2010c:65134)

[15] D. Donatelli and P. Marcati, Convergence of singular limits for multi-D semilinear hyperbolic systems to parabolic systems, Trans. of Amer. Math. Soc. 356, 2093-2121 (2004). MR2031055 (2004k:35240)

[16] P. Marcati and B. Rubino, Hyperbolic to parabolic relaxation theory for quasilinear first order systems, J. Differential Equations 162, 359-399 (2000). MR.1751710 (2001d:35125)

[17] B. Dubroca, J.-L. Feugeas, Entropic moment closure hierarchy for the radiative transfer Equation, C. R. Acad. Sci. Paris, Ser. I, 329, 915-920 (1999). MR1728008 (2000h:85005)

[18] L. Gosse, G. Toscani, Asymptotic-preserving well-balanced scheme for the hyperbolic heat equations, C. R. Acad. Sci. Paris, 334, 337-342 (2002). MR1891014 (2003b:65087)

[19] L. Gosse, G. Toscani, Space localization and well-balanced schemes for discrete kinetic models in diffusive regimes, SIAM J. Numer. Anal., 41, 641-658 (2003). MR2004192 (2004g:65107)

[20] T. Goudon, J.-E. Morel, B. Desprès, C. Buet, C. Berthon, J. Dubois, R. Turpault, J.-F. Coulombel, Mathematical models and numerical methods for radiative transfer, Panoramas et synthèses, Vol. 28, Soc. Française Math., 2009. MR2605788 (2011c:85004)

[21] A. Harten, P.D. Lax, B. van Leer, On upstream differencing and Godunov-type schemes for hyperbolic conservation laws, SIAM Review, 25, 35-61 (1983). MR693713 (85h:65188)

[22] S. Jin, Z. Xin, The relaxation scheme for systems of conservation laws in arbitrary space dimension, Comm. Pure Appl. Math., 45, 235-276 (1995). MR1322811(96c:65134)

[23] N. Ju, Numerical analysis of parabolic $p$-Laplacian: approximation of trajectories. SIAM J. Numer. Anal. 37, 1861-1884 (2000). MR1766851 (2001g:65121)

[24] R. J. LeVeque, Finite volume methods for hyperbolic problems, Cambridge Texts in Applied Mathematics, Cambridge Univ. Press, 2002. MR1925043 (2003h:65001)

[25] T.P. Liu, Hyperbolic conservation laws with relaxation, Comm. Math. Phys., 108, 153-175 (1987). MR872145 (88f:35092)

[26] P. Marcati, Approximate solutions to conservation laws via convective parabolic equations, Comm. Partial Differential Equations, 13, 321-344 (1988). MR917603 (89e:35094)

[27] P. Marcati, A. Milani, The one-dimensional Darcy's law as the limit of a compressible Euler flow, Journal of Differential Equations, 84, no. 1, 129-146 (1990). MR1042662 (91i:35156)

[28] F. Marche, Derivation of a new two-dimensional viscous shallow water model with varying topography, bottom friction and capillary effects, Eur. J. Mech./B-Fluid, 26, 49-63 (2007). MR2281291 (2008d:76026)

[29] G.N. Minerbo, Maximum entropy Eddington factors, J. Quant. Spectrosc. Radiat. Transfer, 20, 541-545 (1978).

[30] I. Suliciu, On modelling phase transitions by means of rate-type constitutive equations, shock wave structure, Int. J. Engrg. Sci. 28, 829-841 (1990). MR1067811(91e:73054)

[31] I. Suliciu, On the thermodynamics of fluids with relaxation and phase transitions. Fluids with relaxation, Int. J. Engag. Sci. 36, 921-947 (1998). MR1636396 (99f:76009)

[32] E.F. Toro, Riemann solvers and numerical methods for fluid dynamics. A practical introduction, Second edition, Springer-Verlag, Berlin, 1999. MR.1717819 (2000f:76091) 
[33] J.L. Vazquez, Hyperbolic aspects in the theory of the porous medium equation, in Proceedings of the Workshop on Metastability and PDE's, Academic Press, Minneapolis, 1985. MR870025 (88f:76037)

[34] W.-A. Yong, Singular perturbations of first-order hyperbolic systems with stiff source terms, J. Differential Equations, 155, 89-132 (1999). MR1693210(2000c:35011)

[35] W.-A. Yong, K. Zumbrun, Existence of relaxation shock profiles for hyperbolic conservation laws, SIAM J. Appl. Math., 60, 1565-1575 (2000). MR1761762 (2001b:35208)

Université de Nantes, Laboratoire de Mathématiques Jean Leray, CNRS UMR 6629, 2 Rue de la Houssinière, BP 92208, 44322 Nantes, France

E-mail address: Christophe.Berthon@math.univ-nantes.fr

Laboratoire Jacques-Louis Lions, Centre National de la Recherche Scientifique, Université Pierre et Marie Curie (Paris 6), 4 Place Jussieu, 75252 Paris, France

E-mail address: contact@philippelefloch.org

Université de Nantes, Laboratoire de Mathématiques Jean Leray, CNRS UMR 6629, 2 RUE DE LA Houssinière, BP 92208, 44322 NAntes, France

E-mail address: Rodolphe.Turpault@univ-nantes.fr 